Fluid Dynamics Research (2008), accepted on 1 December 2007.

\title{
Variational formulation of ideal fluid flows according to gauge principle
}

\author{
Tsutomu Kambe* \\ IDS, Higashi-yama 2-11-3, Meguro-ku, Tokyo 153-0043, Japan
}

Received 6 February 2007; revised 23 November 2007; accepted 1 December 2007

Communicated by Y. Fukumoto.

\begin{abstract}
On the basis of the gauge principle of field theory, a new variational formulation is presented for flows of an ideal fluid. The fluid is defined thermodynamically by mass density and entropy density, and its flow fields are characterized by symmetries of translation and rotation. The rotational transformations are regarded as gauge transformations as well as the translational ones. In addition to the Lagrangians representing the translation symmetry, a structure of rotation symmetry is equipped with a Lagrangian $\Lambda_{A}$ including the vorticity and a vector potential bilinearly. Euler's equation of motion is derived from variations according to the action principle. In addition, the equations of continuity and entropy are derived from the variations. Equations of conserved currents are deduced as the Noether theorem in the space of Lagrangian coordinate $\boldsymbol{a}$. Without $\Lambda_{A}$, the action principle results in the Clebsch solution with vanishing helicity. The Lagrangian $\Lambda_{A}$ yields non-vanishing vorticity and provides a source term of non-vanishing helicity. The vorticity equation is derived as an equation of the gauge field, and the $\Lambda_{A}$ characterizes topology of the field. The present formulation is comprehensive and provides a consistent basis for a unique transformation between the Lagrangian $\boldsymbol{a}$ space and the Eulerian $\boldsymbol{x}$ space. In contrast, with translation symmetry alone, there is an arbitrariness in the transformation between these spaces.
\end{abstract}

Key words: Gauge priciple, Variational formulation, Ideal fluid, Rotation symmetry, Chern-Simons term

* kambe@ruby.dti.ne.jp, http://www.purple.dti.ne.jp/kambe/

Preprint submitted to Elsevier Science 31 October 2018 


\section{Introduction}

A guiding principle in physics is that physical laws should be expressed in a form that is independent of any particular coordinate system. Fluid mechanics is a field theory of Newtonian mechanics that has Galilean symmetry: that is to say, it is covariant under transformations of the Galilei group. Two symmetries (i.e. transformation invariances) are known as subgroups of the Galilei group: translation (space and time) and space-rotation. In the present formulation of flows of an ideal fluid, we seek a scenario which has a formal equivalence with the gauge theory in physics. The gauge theory provides a basis for reflection on the similarity between fluid mechanics and other physical fields.

The gauge principle 1 requires a physical system under investigation to have a symmetry, i.e. a gauge invariance (an invariance with respect to a certain group of transformations). Following this principle, an attempt has been made in Kambe (2007) to study a gauge symmetry of flow fields with respect to translational transformations. The formulation started from a Galilei-invariant Lagrangian of a system of point masses which is known to have global gauge symmetries with respect to both translation and rotation. 2 It was then extended to flows of a fluid, a continuous material characterized with mass density and entropy density. In addition to the global symmetry, local gauge invariance of a Lagrangian is required for such a continuous field. Thus, the convective derivative of fluid mechanics (i.e. the Lagrange derivative) is identified as a covariant derivative, which is a building block in the framework of gauge theory. Based on this, appropriate Lagrangian functionals are defined for motion of an ideal fluid. Euler's equation of motion is derived from the action principle. It is found that a general solution of this formulation is equivalent to the classical Clebsch solution (Clebsch 1859; Lamb 1932), in which the vorticity has a particular form and the helicity vanishes.

In most traditional formulations, the continuity equation and isentropic condition are taken into account as constraints for the variations of the action principle by using Lagrange multipliers, where the isentropy means that each fluid particle of an ideal fluid keeps its entropy value along its trajectory (but the fluid is not necessarily homentropi 3 .) In the present formulation however, those equations are derived from the action principle. The total Lagrangian density consists of kinetic energy and internal energy (with negative sign), supplemented with additional two terms associated with translation invariances

1 See Appendix, or standard texts such as Weinberg (1995), Frankel (1997) or Aitchison \& Hey (1982).

2 These symmetries are interpreted as the homegeneity and isotropy of space in $\S 7$ and 9 of Landau \& Lifshitz (1976). See $\S 2.1$ and Appendix for the definition of global gauge symmetry (i.e. global gauge invariance) and local gauge symmetry.

3 A fluid is homentropic if its entropy per unit mass is uniform throughout space. 
of mass and entropy (see (27) of $\S 3.1$ ). As is interpreted in detail in $\S 4.1$, it is remarkable that the Lagrangians associated with the latter two terms are given such forms as can be eliminated from the action represented with the particle coordinate $\boldsymbol{a}$ (i.e. Lagrangian coordinate), so that the Euler-Lagrange equations represented by the independent varibales $\boldsymbol{a}=\left(a^{1}, a^{2}, a^{3}\right)$ are uninfluenced by the additional terms. Such a form of Lagrangian has close analogy to the Chern-Simons term in the gauge theory.

Earlier papers (Kambe, 2003a, b) investigated the rotation symmetry of the velocity field $\boldsymbol{v}(\boldsymbol{x})$ in a preliminary way, and found that the vorticity $\boldsymbol{\omega}=\nabla \times \boldsymbol{v}$ is in fact a gauge field associated with the rotation symmetry. However in the previous studies including Kambe (2007), the vorticity and helicity were not taken into account in satisfactory manner. Present formulation tries to account for the vorticity and helicity more properly and comprehensively. Thus, the main theme of the present paper is the rotation symmetry.

A new structure of the rotation symmetry is given in $\S 6$ (after a review of the rotational transformations in $\S 5$ ) by the following Lagrangian $\Lambda_{A}$ :

$$
\Lambda_{A}=\int_{M}\left\langle\boldsymbol{A}, E_{W}[\boldsymbol{\omega}]\right\rangle \mathrm{d}^{3} \boldsymbol{x}
$$

where $\boldsymbol{A}$ is a vector potential and $E_{W}[\boldsymbol{\omega}] \equiv \partial_{t} \boldsymbol{\omega}+(\boldsymbol{v} \cdot \nabla) \boldsymbol{\omega}-(\boldsymbol{\omega} \cdot \nabla) \boldsymbol{v}+(\nabla \cdot \boldsymbol{v}) \boldsymbol{\omega}$. This is derived from a Lagrangian form characteristic of a topological term known in the gauge theory. This new term yields non-vanishing rotational component of the velocity field, and provides a source term of non-vanishing helicity. This is also the Chern-Simons term, describing non-trivial topology of vorticity field, i.e. mutual linking of vorticity lines. The vorticity equation is derived as an equation for the gauge field. These are described in $\S 6$ (Lagrangian $L_{A}$ associated with rotation symmetry), $\S 7$ (Corrections to the Variation), and $\S 8$ (Significance of $\Lambda_{A}$ ).

With regard to the variational formulation of fluid flows, the works of Eckart (1938, 1960) and Herivel (1955) are among the earliest to have influenced current formulations. Their variations are carried out in two ways: i.e. a Lagrangian approach and an Eulerian approach. In both approaches, the equation of continuity and the condition of is entropy are added as constraint conditions on the variations by means of Lagrange multipliers. The Lagrangian approach is also taken by Soper (1976). In this relativistic formulation those equation are derived from the equations of current conservation. Several action principles to describe relativistic fluid dynamics have appeared in the past (see Soper $(1976, \S 4.2)$ for some list of them), not only that of Taub (1954).

Firstly, in the Lagrangian approach, the Euler-Lagrange equation results in an equation equivalent to Euler's equation of motion in which the acceleration term is represented as the second time derivative of position coordinates of 
the Lagrangian representation. In this formulation, however, there is a certain degree of freedom in the relation between the Lagrangian particle coordinates $\left(a^{1}, a^{2}, a^{3}\right)$ and Eulerian space coordinates $\left(x^{1}, x^{2}, x^{3}\right)$. Namely, the relation between them is determined only up to an unknown rotation. This point has not been considered seriously in the past, although it is fundamental for the formualtion of flows. This will be discussed more precisely in the sections 4.2 and 8.2. A common feature of Herivel (1955) and Eckart (1960) is that both arrive at the Clebsch solution in the Eulerian representaion (see Sec.A and 3.2). However, it was remarked by Bretherton (1970) that the Clebsch representation has only local validity in the neighbourhood of a chosen point if vortex lines are knotted or linked.

In the second approach, referred to as the Eulerian description, the action principle of an ideal fluid results only in potential flows if the fluid is homentropic, as shown in Kambe (2003a, 2007). However it is well-known that, even in such a homentropic fluid, it should be possible to have rotational flows 4 This is a long-standing problem (Serrin 1959; Lin 1963; Seliger \& Whitham 1968; Bretherton 1970; Salmon 1988). Lin (1963) tried to resolve this difficulty by introducing a constraint as a side condition, imposing invariance of Lagrangian particle labels $\left(a^{1}, a^{2}, a^{3}\right)$ along particle trajectories. The constraints for the variation are defined by using Lagrange multipliers (functions of positions), which are called 'potentials'. In addition, the continuity equation and isentropic condition are also taken into account by using Lagrange multipliers. However, the physical significance of those potentials introduced as the Lagrange multipliers is not clear. Mysteriously, the Lagrange multiplier for the continuity equation becomes the velocity potential for the irrotational part of the velocity. Gauge theory for fluid flows provides a crucial key to resolve these issues, which is the main target of the present investigation.

Symmetries imply conservation laws. According to the relativistic formulation (e.g. Soper 1976; Landau \& Lifshitz 1987 (Ch.XV)), as long as the flow fields obey the Euler-Lagrange equation, an energy-momentum tensor $T^{\mu \nu}$ must obey the conservation equation of the form $\partial_{\nu} T^{\mu \nu}=0$ (considered in Sec. 4.2 below). The Energy-momentum tensor $T^{\mu \nu}$ in the present case is given in Sec.4. A similar field-theoretic approach is taken in Jackiw (2002) by applying the ideas of particle physics to fluid mechanics in terms of Hamiltonian and Poisson brackets, both relativistically and nonrelativistically, and extension to supersymmetry is also considered. In Soper (1976) and Jackiw (2002), the nonrelativistic part follows the traditional approach and no explicit gaugetheoretic consideration is given to fluid mechanics in the sense of the present

4 In fact, Euler (1755) showed that his equation of motion can drescribe rotational flows, in the historical paper: L. Euler, Principes généraux du mouvement des fluides, MASB, v. 11 (1757), 274-315. (MASB: Memoires de l'Académie Ryale des Sciences, année 1755. Berlin 1757.) 
formulation.

The appendix describes the background of the present theory: (A) Known gauge invariances in two systems of electromagnetism and Clebsch form of fluid flows, (B) Related aspects in quantum mechanics and relativistic theory, and $(\mathrm{C})$ Brief scenario of gauge principle.

\section{Translation symmetry of flow fields}

The formulation of Kambe (2007) started from a Galilei-invariant Lagrangian of a system of point masses, which is known to have global gauge symmetries with respect to both translation and rotation. Concept of local transformation is an extention of the global transformation to describe a continuous field. In order to represent a continuous distribution of mass, the discrete positions of point masses are replaced by continuous parameters $\boldsymbol{a}=\left(a^{1}, a^{2}, a^{3}\right)$ of particles in a bounded sub-space $M$ (of consideration) in the three-dimensional Euclidean space $E^{3}$. The spatial position of each mass particle labelled by $\boldsymbol{a}$ (Lagrange parameter) is denoted by $\boldsymbol{x}=X_{a}(t) \equiv X(\boldsymbol{a}, t)$, a function of $\boldsymbol{a}$ as well as the time $t$. An Eulerian space point is denoted by $\boldsymbol{x}=\left(x^{1}, x^{2}, x^{3}\right)$. The fluid particle occupying the point $\boldsymbol{x}$ at time $t$ is denoted by $\boldsymbol{a}(\boldsymbol{x}, t) 5$

\subsection{Global invariance of a fluid}

For a continuous distribution of mass (i.e. for a fluid), Lagrangian functional is defined as

$$
\Lambda=\int L(x, v) \mathrm{d}^{3} x,
$$

where $x$ and $v$ denote $X_{a}^{k}(t)$ and $v^{k}=\partial_{t} X_{a}^{k}(t)(k=1,2,3)$ respectively, and $L(x, v)$ is a Lagrangian density. Suppose that an infinitesimal transformation is expressed by $x \rightarrow x^{\prime}=x+\delta x$ and $v \rightarrow v^{\prime}=v+\delta v$, where $\delta x=\xi(x, t)$ and $\delta v=\partial_{t}(\delta x)$, a component of $\xi$ is denoted by $\xi^{k}(x, t)$, an arbitrary differentiable variation field. The resulting variation of the Lagrangian density $L(x, v)$ is

$$
\delta L=\left(\frac{\partial L}{\partial x^{k}}-\partial_{t}\left(\frac{\partial L}{\partial v^{k}}\right)\right) \delta x^{k}+\partial_{t}\left(\frac{\partial L}{\partial v^{k}} \delta x^{k}\right)
$$

$\overline{5}$ Here the Lagrangian coordinates $\boldsymbol{a}=\left(a^{1}, a^{2}, a^{3}\right)$ are defined so as to represent the mass coordinate such that $\mathrm{d}^{3} \boldsymbol{a}=\rho \mathrm{d}^{3} \boldsymbol{x}$, where $\mathrm{d}^{3} \boldsymbol{a}=\mathrm{d} a^{1} \mathrm{~d} a^{2} \mathrm{~d} a^{3}$ and $\mathrm{d}^{3} \boldsymbol{x}=$ $\mathrm{d} x^{1} \mathrm{~d} x^{2} \mathrm{~d} x^{3}$. A variable such as $X_{a}$ denotes the one with respect to the particle $\boldsymbol{a}$. 
This does not vanish in general. In fact, assuming the Euler-Lagrange equation, $\partial L / \partial x^{k}-\partial_{t}\left(\partial L / \partial v^{k}\right)=0$, we obtain

$$
\delta L=\partial_{t}\left(\frac{\partial L}{\partial v^{k}} \delta x^{k}\right)=\partial_{t}\left(\frac{\partial L}{\partial v^{k}}\right) \xi^{k}+\frac{\partial L}{\partial v^{k}} \partial_{t} \xi^{k} .
$$

Global invariance, i.e. $\delta \Lambda=\int \delta L(x, v) \mathrm{d}^{3} x=0$ for arbitrary constants $\xi^{k}$ $(k=1,2,3)$, requires that the total momentum defined by $\int\left(\partial L / \partial v^{k}\right) \mathrm{d}^{3} x$ must be invariant. In the local transformation, however, the variation field $\xi^{k}$ depends on the time $t$ and space point $x$, and the variation $\delta \Lambda=\int \delta L \mathrm{~d}^{3} x$ does not vanish in general.

\subsection{Covariant derivative}

According to the gauge principle of Appendix $\mathrm{C}$, non-vanishing of $\delta \Lambda$ is understood as meaning that a new field $G$ must be taken into account in order to achieve local gauge invariance of the Lagrangian $\Lambda$. To that end, we try to replace the partial time derivative $\partial_{t}$ by a covariant derivative $\mathrm{D}_{t}$, where the derivative $\mathrm{D}_{t}$ is defined by $\mathrm{D}_{t}:=\partial_{t}+G$. In dynamical systems like the present one, the time derivative is the primary object to be considered in the analysis of local gauge transformation, as implied by the expression (2). Thus, the time derivatives $\partial_{t} \xi$ and $\partial_{t} q$ are replaced by $\mathrm{D}_{t} \xi=\partial_{t} \xi+G \xi$ and $\mathrm{D}_{t} q=\partial_{t} q+G q$, where $q$ is understood to denote $\boldsymbol{x}$. A free-field Lagrangian $\Lambda_{\mathrm{f}}$ is defined by

$$
\Lambda_{\mathrm{f}}=\int L_{\mathrm{f}}(v) \mathrm{d}^{3} \boldsymbol{x}:=\frac{1}{2} \int\langle v, v\rangle \rho \mathrm{d}^{3} x=\frac{1}{2} \int\left\langle\mathrm{D}_{t} q, \mathrm{D}_{t} q\right\rangle \mathrm{d}^{3} \boldsymbol{a},
$$

where $\rho \mathrm{d}^{3} x \equiv \mathrm{d}^{3} \boldsymbol{a}$ denotes the mass element in a volume element $\mathrm{d}^{3} \boldsymbol{x}$ of the $x$-space with $\rho$ the mass-density, and $v=\mathrm{D}_{t} q$.

\subsection{Galilean transformation}

\subsubsection{Global transformation}

Symmetries to be investigated are the translation symmetry and rotation symmetry, which exist in the discrete system. The Lagrangian $\Lambda_{\mathrm{f}}$ defined by (3) for a continuous field has such global symmetries, inherited from the discrete system. It is a primary concern here to investigate whether the system of fluid flows satisfies local invariance. We consider the translation symmetry first, and in the second half of the present analysis we investigate the rotation symmetry.

A translational transformation from one frame $F$ to another $F^{\prime}$ moving with a relative velocity $\boldsymbol{U}$ is a Galilean transformation in Newtonian mechanics. 
The transformation is defined by

$$
x \equiv(t, \boldsymbol{x}) \Rightarrow x^{\prime} \equiv\left(t^{\prime}, \boldsymbol{x}^{\prime}\right)=(t, \boldsymbol{x}-\boldsymbol{U} t)
$$

This is a sequence of global translations with a parameter $t$. Corresponding transformation of velocity is as follows: $v \equiv(1, \boldsymbol{v}) \Rightarrow v^{\prime} \equiv\left(1, \boldsymbol{v}^{\prime}\right)=$ $(1, \boldsymbol{v}-\boldsymbol{U})$. From (4), differential operators $\partial_{t}=\partial / \partial t$ and $\partial_{k}=\partial / \partial x_{k}\left(\partial_{k}^{\prime}=\right.$ $\left.\partial / \partial x_{k}^{\prime}\right)$ are transformed according to $\partial_{t}=\partial_{t^{\prime}}-\boldsymbol{U} \cdot \boldsymbol{\nabla}^{\prime}$ and $\boldsymbol{\nabla}=\boldsymbol{\nabla}^{\prime}$, where $\boldsymbol{\nabla}=\left(\partial_{1}, \partial_{2}, \partial_{3}\right)$ and $\boldsymbol{\nabla}^{\prime}=\left(\partial_{1}^{\prime}, \partial_{2}^{\prime}, \partial_{3}^{\prime}\right)$. There is a transformation invariance of the convective derivative $\mathrm{D}_{c} \equiv \partial_{t}+(\boldsymbol{v} \cdot \boldsymbol{\nabla})$ : namely $\partial_{t}+(\boldsymbol{v} \cdot \boldsymbol{\nabla})=\partial_{t^{\prime}}+\left(\boldsymbol{v}^{\prime} \cdot \boldsymbol{\nabla}^{\prime}\right)$.

\subsubsection{Local Galilean transformation}

The velocity field $\boldsymbol{v}(\boldsymbol{x}, t)$ is defined by the particle velocity, i.e.

$$
\boldsymbol{v}\left(X_{a}, t\right)=\frac{\mathrm{d}}{\mathrm{d} t} X_{a}(t)
$$

Consider the following infinitesimal transformation:

$$
\boldsymbol{x}^{\prime}(\boldsymbol{x}, t)=\boldsymbol{x}+\boldsymbol{\xi}(\boldsymbol{x}, t), \quad t^{\prime}=t .
$$

This may be regarded as a local gauge transformation between non-inertial frames. In fact, the transformations (6) is understood to mean that the coordinate $\boldsymbol{x}$ of a fluid particle at $\boldsymbol{x}=X_{a}(t)$ in the frame $F$ is transformed to the new coordinate $\boldsymbol{x}^{\prime}$ of $F^{\prime}$, given by $\boldsymbol{x}^{\prime}=X_{a}^{\prime}\left(X_{a}, t\right)=X_{a}(t)+\boldsymbol{\xi}\left(X_{a}, t\right)$. Therefore, its velocity $\boldsymbol{v}=(\mathrm{d} / \mathrm{d} t) X_{a}(t)$ is transformed by

$$
\begin{aligned}
\boldsymbol{v}^{\prime}\left(\boldsymbol{x}^{\prime}\right) & \equiv \frac{\mathrm{d}}{\mathrm{d} t} X_{a}^{\prime}=\frac{\mathrm{d}}{\mathrm{d} t}\left(X_{a}(t)+\boldsymbol{\xi}\left(X_{a}, t\right)\right)=\boldsymbol{v}\left(X_{a}\right)+(\mathrm{d} / \mathrm{d} t) \boldsymbol{\xi}_{a}, \\
(\mathrm{~d} / \mathrm{d} t) \boldsymbol{\xi}_{a} & =\partial_{t} \boldsymbol{\xi}+\left.(\boldsymbol{v} \cdot \nabla) \boldsymbol{\xi}\right|_{\boldsymbol{x}_{X_{a}}}, \quad \boldsymbol{\xi}_{a} \equiv \boldsymbol{\xi}\left(X_{a}, t\right) .
\end{aligned}
$$

This is interpreted as follows: the origin of a local frame is displaced by $-\boldsymbol{\xi}$ where its coordinate axes are moving (without rotation) with the velocity $-(\mathrm{d} / \mathrm{d} t) \boldsymbol{\xi}$ in accelerating motion (non-inertial frame). This implies that the velocity $\boldsymbol{v}(\boldsymbol{x})$ is transformed according to (7), since the local frame is moving with the velocity $-(\mathrm{d} / \mathrm{d} t) \boldsymbol{\xi}$. Note that, in this transformation, the points $\boldsymbol{x}$ and $\boldsymbol{x}^{\prime}$ are the same with respect to the space $F$. In view of the transformation (6), the time derivative and spatial derivatives are transformed as

$$
\begin{aligned}
\partial_{t} & =\partial_{t^{\prime}}+\left(\partial_{t} \boldsymbol{\xi}\right) \cdot \nabla^{\prime}, & \nabla^{\prime} & =\left(\partial_{k}^{\prime}\right), \\
\partial_{k} & =\partial_{k}^{\prime}+\partial_{k} \xi_{l} \partial_{l}^{\prime}, & \partial_{k}^{\prime} & =\partial / \partial x_{k}^{\prime} .
\end{aligned}
$$




\subsection{Invariance of $D_{t}$ and definition of velocity}

In this local transformation too, there is an invariance of the convective derivative $\mathrm{D}_{c} \equiv \partial_{t}+(\boldsymbol{v} \cdot \boldsymbol{\nabla})=\partial_{t^{\prime}}+\left(\boldsymbol{v}^{\prime} \cdot \boldsymbol{\nabla}^{\prime}\right)$. In fact, from (10) and (7), we obtain

$$
\boldsymbol{v} \cdot \boldsymbol{\nabla}=\boldsymbol{v} \cdot \boldsymbol{\nabla}^{\prime}+(\boldsymbol{v} \cdot \boldsymbol{\nabla} \xi) \cdot \boldsymbol{\nabla}^{\prime}=\boldsymbol{v}^{\prime}\left(\boldsymbol{x}^{\prime}\right) \cdot \boldsymbol{\nabla}^{\prime}+(-(\mathrm{d} \boldsymbol{\xi} / \mathrm{d} t)+\boldsymbol{v} \cdot \boldsymbol{\nabla} \xi) \cdot \nabla^{\prime},
$$

where $\boldsymbol{v}=\boldsymbol{v}^{\prime}-\mathrm{d} \boldsymbol{\xi} / \mathrm{d} t$. The last term is $-\partial_{t} \boldsymbol{\xi} \cdot \nabla^{\prime}$ by (8). Thus, we find the invariance of $\mathrm{D}_{c}$ by (9). This invariance (i.e. the transformation symmetry) implies that the covariant derivative $\mathrm{D}_{t}$ is in fact given by the convective derivative $\mathrm{D}_{c}$ :

$$
\mathrm{D}_{t} \equiv \partial_{t}+(\boldsymbol{v} \cdot \boldsymbol{\nabla})=\partial_{t^{\prime}}+\left(\boldsymbol{v}^{\prime} \cdot \nabla^{\prime}\right)
$$

(see Kambe (2007) for its direct derivation).

For a scalar function $f(\boldsymbol{x}, t)$, we have $\mathrm{D}_{t} f=\partial_{t} f+\boldsymbol{v} \cdot \nabla f$ (Lagrange derivative of $f(\boldsymbol{x}, t))$. It is important to recognize that this derivative $\mathrm{D}_{t} f$ has an intrinsic meaning. That is, we have the invariance: $\mathrm{D}_{t} f=\mathrm{D}_{t^{\prime}}^{\prime} f^{\prime}$, with respect to the local Galilean transformation (6), since a scalar means $f^{\prime} \equiv f^{\prime}\left(\boldsymbol{x}^{\prime}, t^{\prime}\right)=f(\boldsymbol{x}, t)$. In general, vectors or tensors need not have this property.

By using the covariant derivative $\mathrm{D}_{t}$, we can define the velocity $\boldsymbol{v}$ by $\mathrm{D}_{t} \boldsymbol{x}$. In fact, each component $a^{i}$ of the particle label $\boldsymbol{a}$ is a scalar, and satisfies $\mathrm{D}_{t} a^{i}=\partial_{t} a^{i}+(\boldsymbol{v} \cdot \nabla) a^{i}=0$, since the particle with the label $a^{i}$ moves with the velocity $\boldsymbol{v}=\partial_{t} X(\boldsymbol{a}, t)$ by the definition (5). Setting $\boldsymbol{x}=X(\boldsymbol{a}, t)$ for the particle position, we have

$$
\mathrm{D}_{t} X(\boldsymbol{a}, t)=\partial_{t} X(\boldsymbol{a}, t)+\mathrm{D}_{t} \boldsymbol{a} \cdot \nabla_{a} X=\partial_{t} X(\boldsymbol{a}, t)=\boldsymbol{v},
$$

where $\left(\nabla_{a} \boldsymbol{X}\right)=\left(\partial X^{k} / \partial a^{l}\right)$. In addition, applying $\mathrm{D}_{t}$ to (6) and using $\mathrm{D}_{t^{\prime}}^{\prime}=$ $\mathrm{D}_{t}$, we have $\boldsymbol{v}^{\prime}=\mathrm{D}_{t^{\prime}}^{\prime} \boldsymbol{x}^{\prime}=\mathrm{D}_{t}(\boldsymbol{x}+\boldsymbol{\xi})=\boldsymbol{v}+\mathrm{D}_{t} \boldsymbol{\xi}$ (consistent with (7)). Thus, the particle velocity $\boldsymbol{v}$ can be defined by $\boldsymbol{v}(\boldsymbol{x}, t)=\mathrm{D}_{t} \boldsymbol{x}$.

\subsection{Transformation of acceleration}

The gauge transformation of Sec.2.3.2 defines the transformations: $\boldsymbol{x} \rightarrow \boldsymbol{x}^{\prime}=$ $\boldsymbol{x}+\boldsymbol{\xi}$ and $\boldsymbol{v}(\boldsymbol{x}) \rightarrow \boldsymbol{v}^{\prime}\left(\boldsymbol{x}^{\prime}\right)=\boldsymbol{v}(\boldsymbol{x})+\mathrm{D}_{t} \boldsymbol{\xi}$. Namely, the variations of $x^{i}$ and $v^{i}$ are given by

$$
\begin{aligned}
& \delta x^{i}=\xi^{k} T_{k} x^{i}=\xi^{i}, \quad\left(T_{k}=\partial / \partial x^{k}\right) \\
& \delta v^{i}=\mathrm{D}_{t} \xi^{i}=\partial_{t} \xi^{i}+v^{k} T_{k} \xi^{i},
\end{aligned}
$$


where $T_{k} x^{i}=\delta_{k}^{i}$. Next, let us consider acceleration of a fluid particle. Differentiating (7) with respect to $t$ again, we obtain

$$
\frac{\mathrm{d}^{2}}{\mathrm{~d} t^{2}} X_{a}^{\prime}=\frac{\mathrm{d}^{2}}{\mathrm{~d} t^{2}} X_{a}(t)+\frac{\mathrm{d}^{2}}{\mathrm{~d} t^{2}} \boldsymbol{\xi}\left(X_{a}, t\right)
$$

This describes a relation between accelerations in the two frames $F$ and $F^{\prime}$. Let us define the covariant derivative of the velocity $v=\left(v^{i}\right)$ by $\mathrm{D}_{t} v=\partial_{t} v+v^{k} T_{k} v$. Variation of $\partial_{t} v$ is given by

$$
\delta\left(\partial_{t} v\right)=\partial_{t}(\delta v)+\left(\delta \partial_{t}\right) v=\partial_{t}\left(\mathrm{D}_{t} \xi\right)-\left(\partial_{t} \xi^{k} T_{k}\right) v
$$

where $\delta \partial_{t}=\partial_{t^{\prime}}-\partial_{t}=-\partial_{t} \xi^{k} T_{k}$ from (9) (to the first order of $\xi$ ). Then, using $\delta v=\mathrm{D}_{t} \xi$ and $\delta T_{k}=T_{k}^{\prime}-T_{k}=-\left(T_{k} \xi^{l}\right) T_{l}$ from (10), the transformation of $\mathrm{D}_{t} v$ is given by $\delta\left(\mathrm{D}_{t} v\right)=\delta\left(\partial_{t} v\right)+\left(\delta v^{k}\right) T_{k} v+v^{k}\left(\delta T_{k}\right) v+v^{k} T_{k}(\delta v)$, namely,

$$
\delta\left(\mathrm{D}_{t} v\right)=\partial_{t}\left(\mathrm{D}_{t} \xi\right)-\partial_{t} \xi^{k} T_{k} v+\left(\mathrm{D}_{t} \xi^{k}\right) T_{k} v-v^{k}\left(T_{k} \xi^{l}\right) T_{l} v+v^{k} T_{k}\left(\mathrm{D}_{t} \xi\right) .
$$

Thus, we obtain

$$
\delta\left(\mathrm{D}_{t} v\right)=\mathrm{D}_{t}\left(\mathrm{D}_{t} \xi\right), \quad \mathrm{D}_{t}=\partial_{t}+v^{k} T_{k}
$$

It is found that the covariant derivative $\mathrm{D}_{t} v$ transforms just like the acceleration $\mathcal{A}=(\mathrm{d} / \mathrm{d} t)^{2} X_{a}(t)$ according to (15). This implies that $\mathrm{D}_{t} v$ represents the particle acceleration $\mathcal{A}(\boldsymbol{x}, t)$ :

$$
\mathcal{A}=\mathrm{D}_{t} \boldsymbol{v}=\left(\partial_{t}+v^{k} \partial_{k}\right) \boldsymbol{v}
$$

\subsection{Galilean invariant Lagrangian}

According to the formulation of Kambe (2007) based on the gauge principle, the total Lagrangian is defined by

$$
\Lambda_{\mathrm{T}}=\Lambda_{\mathrm{f}}+\Lambda_{\epsilon}+\Lambda_{\phi}+\Lambda_{\psi}
$$

as far as the translation symmetry is concerned, where

$$
\begin{array}{ll}
\Lambda_{\mathrm{f}}=\int \frac{1}{2}\langle\boldsymbol{v}(x), \boldsymbol{v}(x)\rangle \rho \mathrm{d}^{3} \boldsymbol{x}, & \Lambda_{\epsilon}=-\int_{M} \epsilon(\rho, s) \rho \mathrm{d}^{3} \boldsymbol{x}, \\
\Lambda_{\phi}=-\int_{M}\left(\mathrm{D}_{t} \phi\right) \rho \mathrm{d}^{3} \boldsymbol{x}, & \Lambda_{\psi}=-\int_{M}\left(\mathrm{D}_{t} \psi\right) \rho s \mathrm{~d}^{3} \boldsymbol{x} .
\end{array}
$$

The variables $\boldsymbol{v}, \rho, \epsilon$ and $s$ are the velocity vector, density, internal energy (per unit mass) and entropy (per unit mass) of the fluid, $\phi(\boldsymbol{x}, t)$ and $\psi(\boldsymbol{x}, t)$ are gauge potentials associated with the mass density $\rho$ and entropy density $\rho s$ respectively. The velocity is defined by $\boldsymbol{v}(\boldsymbol{x}, t)=\mathrm{D}_{t} \boldsymbol{x}$, where $\mathrm{D}_{t}=\partial_{t}+\boldsymbol{v} \cdot \nabla$. 
From $\S 2.4$, it is almost obvious that the two Lagrangians $\Lambda_{\phi}$ and $\Lambda_{\psi}$ of (19) are invariant with respect to the local Galilean transformation (i.e. local gauge transformation).

\section{Variational principle: Translation symmetry}

\subsection{Action principle}

The action $I$ and the action principle $\delta I=0$ are defined by

$$
\begin{aligned}
I= & \int_{t_{0}}^{t_{1}} \Lambda_{\mathrm{T}} \mathrm{d} t=\int_{t_{0}}^{t_{1}} \int_{M} \mathrm{~d} t \mathrm{~d}^{3} \boldsymbol{x} L_{\mathrm{T}}[\boldsymbol{v}, \rho, s, \phi, \psi], \\
\delta I= & \delta \int_{t_{0}}^{t_{1}} \int_{M} \mathrm{~d} t \mathrm{~d}^{3} \boldsymbol{x} L_{\mathrm{T}}=0, \\
& L_{\mathrm{T}} \equiv \frac{1}{2} \rho\langle\boldsymbol{v}, \boldsymbol{v}\rangle-\rho \epsilon(\rho, s)-\rho \mathrm{D}_{t} \phi-\rho s \mathrm{D}_{t} \psi .
\end{aligned}
$$

where $L_{\mathrm{T}}$ is the Lagrangian density. There is a certain thermodynamic property that must be taken into consideration. That is the definition of an ideal fluid. Namely, there is no dissipation of kinetic energy into heat, i.e. there is no heat production within the ideal fluid. By thermodynamics, if there is no heat production, we have $T \delta s=0$ ( $T$ : temperature). Then,

$$
\delta \epsilon=(\delta \epsilon)_{s}=\frac{p}{\rho^{2}} \delta \rho, \quad\left(\frac{\partial \epsilon}{\partial \rho}\right)_{s}=\frac{p}{\rho^{2}}, \quad \delta h=\frac{1}{\rho} \delta p
$$

where $p$ is the fluid pressure, $h$ is the enthalpy defined by $\epsilon+p / \rho$, and $(\cdot)_{s}$ denotes $s$ being fixed. However, the entropy $s$ may not be uniform and depend on $\boldsymbol{x}$ through the initial condition.

One of the aims of the present analysis is to show that the previous formulation (Kambe 2007) based on the symmetry of parallel translation alone is not sufficient to represent rotational flows with non-vanishing helicity. The rotaional symmetry will be considered from $\S 5$ below.

\subsection{Outcomes of variations}

Writing the Lagrangian density $(22)$ as $L_{\mathrm{T}}[\boldsymbol{v}, \rho, s, \phi, \psi]$, we take variations of the variables $\boldsymbol{v}, \rho, s$ and potentials $\phi$ and $\psi$, where variations are assumed independent. Substituting the varied variables $\boldsymbol{v}+\delta \boldsymbol{v}, \rho+\delta \rho s+\delta s, \phi+\delta \phi$ and $\psi+\delta \psi$ into $L_{\mathrm{T}}[\boldsymbol{v}, \rho, s, \phi, \psi]$ and writing its variation as $\delta L_{\mathrm{T}}$, we obtain 


$$
\begin{aligned}
\delta L_{\mathrm{T}}= & \delta \boldsymbol{v} \cdot \rho(\boldsymbol{v}-\nabla \phi-s \nabla \psi)-\delta s \rho \mathrm{D}_{t} \psi \\
& +\delta \rho\left(\frac{1}{2} v^{2}-h-\mathrm{D}_{t} \phi-s \mathrm{D}_{t} \psi\right) \\
& +\delta \phi\left(\partial_{t} \rho+\nabla \cdot(\rho \boldsymbol{v})\right)-\partial_{t}(\rho \delta \phi)-\nabla \cdot(\rho \boldsymbol{v} \delta \phi) \\
& +\delta \psi\left(\partial_{t}(\rho s)+\nabla \cdot(\rho s \boldsymbol{v})\right)-\partial_{t}(\rho s \delta \psi)-\nabla \cdot(\rho s \boldsymbol{v} \delta \psi),
\end{aligned}
$$

where $(\partial / \partial \rho)(\rho \epsilon)=\epsilon+\rho(\partial \epsilon / \partial \rho)_{s}=\epsilon+p / \rho=h$ is used. The variation fields are assumed to vanish on the boundary surface enclosing the domain $M$, as well as at both ends $t_{0}$ and $t_{1}$ of time integration of the action $I$ (where $M$ is chosen arbitrarily), as is usually the case. Thus, the action principle $\delta I=0$ for independent variations $\delta \boldsymbol{v}, \delta \rho$ and $\delta s$ results in

$$
\begin{array}{ll}
\delta \boldsymbol{v}: & \boldsymbol{v}=\nabla \phi+s \nabla \psi, \\
\delta \rho: & \frac{1}{2} v^{2}-h-\mathrm{D}_{t} \phi-s \mathrm{D}_{t} \psi=0, \\
\delta s: & \mathrm{D}_{t} \psi \equiv \partial_{t} \psi+\boldsymbol{v} \cdot \nabla \psi=0 .
\end{array}
$$

Using (25) and (27), we have

$$
\mathrm{D}_{t} \phi=\partial_{t} \phi+\boldsymbol{v} \cdot \nabla \phi=\partial_{t} \phi+\boldsymbol{v} \cdot(\boldsymbol{v}-s \nabla \psi)=v^{2}+\partial_{t} \phi+s \partial_{t} \psi .
$$

Under this relation and (27), the equation (26) can be rewritten as

$$
\frac{1}{2} v^{2}+h+\partial_{t} \phi+s \partial_{t} \psi=0 .
$$

This is regarded as an integral of motion. From the variations of $\delta \phi$ and $\delta \psi$,

$$
\begin{array}{ll}
\delta \phi: & \partial_{t} \rho+\nabla \cdot(\rho \boldsymbol{v})=0, \\
\delta \psi: & \partial_{t}(\rho s)+\nabla \cdot(\rho s \boldsymbol{v})=0 .
\end{array}
$$

Using (29), the second equation reduces to the adiabatic equation:

$$
\partial_{t} s+\boldsymbol{v} \cdot \nabla s=\mathrm{D}_{t} s=0 .
$$

Thus, we have obtained the continuity equation (29) and entropy equation (31) from the action principle.

It is shown in Kambe (2007) that the present solution is equivalent to the classical Clebsch solution (Clebsch 1859). With the velocity (25), the vorticity $\boldsymbol{\omega}$ is given by

$$
\boldsymbol{\omega}=\nabla \times \boldsymbol{v}=\nabla s \times \nabla \psi .
$$

This implies that the vorticity is connected with non-uniformity of entropy. Using (28), we find that the following Euler's equation of motion is satisfied:

$$
\partial_{t} \boldsymbol{v}+\boldsymbol{\omega} \times \boldsymbol{v}=-\nabla\left(\frac{1}{2} v^{2}+h\right) .
$$


In this case, the helicity vanishes (Bretherton, 1970). For a general velocity field, the helicity $H$ defined by

$$
H[V] \equiv \int_{V} \boldsymbol{\omega} \cdot \boldsymbol{v} \mathrm{d}^{3} \boldsymbol{x}
$$

is a measure of linkage and knottedness of vortex lines, and does not vanish in general (see Sec.8.1).

\subsection{Homentropic fluid}

For a homentropic fluid in which the entropy $s$ is a uniform constant $s_{0}$ at all points, we have $\epsilon=\epsilon(\rho), \mathrm{d} \epsilon=\left(p / \rho^{2}\right) \mathrm{d} \rho$ and $\mathrm{d} h=(1 / \rho) \mathrm{d} p$ from (23) since $\delta s=0$. In addition, the motion is irrotational. In fact, from (25), we have $\boldsymbol{v}=\nabla \Phi$ where $\Phi=\phi+s_{0} \psi$, and $\boldsymbol{\omega}=0$ from (32). The integral (28) becomes $\frac{1}{2} v^{2}+h+\partial_{t} \Phi=0$. The Euler's equation (33) reduces to

$$
\partial_{t} \boldsymbol{v}+\nabla\left(\frac{1}{2} v^{2}\right)=-\nabla h, \quad \text { where } \quad \nabla h=\frac{1}{\rho} \nabla p
$$

Note that the left hand side is the convective time derivative for the irrotational velocity $v^{k}=\partial_{k} \Phi$ :

$$
\partial_{t} \boldsymbol{v}+\nabla\left(\frac{1}{2} v^{2}\right)=\partial_{t} \boldsymbol{v}+(\boldsymbol{v} \cdot \nabla) \boldsymbol{v}=\mathrm{D}_{t} \boldsymbol{v}
$$

Thus, as far as the action principle is concerned for a homentropic fluid, Euler's equation of motion reduces to that for potential flows of a perfect fluid.

Traditionally, this property is considered to be a defect of the formulation of the Eulerian variation described in the previous section, because the action principle should yield the equations for rotational flows as well. In order to remove this (apparent) flaw, Lin (1963) introduced the condition for the conservation of the identity of particles denoted by $\boldsymbol{a}=\left(a^{k}\right)$, which is represented by an additional subsidiary Lagrangian of the form $\int A_{k} \cdot \mathrm{D}_{t} a^{k} \mathrm{~d}^{3} \boldsymbol{x}$. This introduces three potentials $A_{k}(\boldsymbol{x}, t)$ as a set of Lagrange multipliers of conditional variation. Problem is that physical significance of $A_{k}$ is not clear (Bretherton 1970; Seliger \& Whitham 1968; Salmon 1988).

Let us recall that we have been considering the Lagrangian $\Lambda_{\mathrm{T}}$ satisfying the symmetry of parallel translation. However, the flow field has another symmetry of rotational invariance (Kambe 2003a, b). The equation (31) implies that the entropy $s$ plays the role to identify each fluid particle. Furthermore, local rotation is captured by the expression (32). However, in a homentropic fluid, there is no such machinery to identify each fluid particle. Gauge invariance with respect to local rotation could be a candidate instead of $s$. 
Insofar as the flow field is characterized by the translation symmetry alone, we have arrived at the above result, i.e. the flow field should be irrotational if the fluid is homentropic. The fluid motion is driven by the velocity potential $\Phi$, where $\Phi=\phi+s_{0} \psi$ with $\phi$ and $\psi$ the gauge potentials.

\section{Equations in $a$-space}

\subsection{Lagrangian}

Let us consider another variational formulation with the Lagrangian represented by the particle coordinates $\boldsymbol{a}=\left(a^{1}, a^{2}, a^{3}\right)=(a, b, c)$. Independent variables are denoted with $a^{\mu}(\mu=0,1,2,3)$ with $a^{0}$ being the time variable written as $\tau(=t)$. The letter $\tau$ is used instead of $t$ in combination with the particle coordinates $a^{k}(k=1,2,3)$. The physical-space position of a particle is expressed by $X^{k}\left(a^{\mu}\right)$, or $(X, Y, Z)$. Its velocity $v^{k}$ is given by $\partial_{\tau} X^{k}=X_{\tau}^{k}$. The derivative $\partial_{\tau}=\partial / \partial \tau$ is equivalent to the covariant derivative $\mathrm{D}_{t}$ :

$$
\partial_{\tau}=\partial_{t}+u \partial_{x}+v \partial_{y}+w \partial_{z}=\partial_{t}+\boldsymbol{v} \cdot \nabla
$$

Mass density $\rho$ is defined by the relation, $\rho \mathrm{d}^{3} \boldsymbol{x}=\mathrm{d}^{3} \boldsymbol{a}$, where $\mathrm{d}^{3} \boldsymbol{x}$ denotes the volume element $\mathrm{d} X^{1} \mathrm{~d} X^{2} \mathrm{~d} X^{3}$. Using the Jacobian determinant $J$ of the transformation from $\boldsymbol{a}$-space to $\boldsymbol{X}$-space, we obtain

$$
\rho=\frac{1}{J}, \quad J=\frac{\partial\left(X^{1}, X^{2}, X^{3}\right)}{\partial\left(a^{1}, a^{2}, a^{3}\right)}=\frac{\partial(X, Y, Z)}{\partial(a, b, c)} .
$$

The total Lagrangian (17) with (18) and (19) is rewritten as

$$
\Lambda_{\mathrm{T}}=\int \frac{1}{2} X_{\tau}^{k} X_{\tau}^{k} \mathrm{~d}^{3} \boldsymbol{a}-\int \epsilon(\rho, s) \mathrm{d}^{3} \boldsymbol{a}-\int \partial_{\tau} \phi \mathrm{d}^{3} \boldsymbol{a}-\int s \partial_{\tau} \psi \mathrm{d}^{3} \boldsymbol{a} .
$$

The action $I$ is defined by integration of $\Lambda_{\mathrm{T}}$ with respect to the time $\tau$ :

$$
I=\int_{\tau_{1}}^{\tau_{2}} \Lambda_{\mathrm{T}} \mathrm{d} \tau
$$

In this definition, the third integral $I_{3}=\int \mathrm{d} \tau \int \partial_{\tau} \phi \mathrm{d}^{3} \boldsymbol{a}$ can be integrated with respect to $\tau$, and expressed as $\int[\phi] \mathrm{d}^{3} \boldsymbol{a}$, where $[\phi]=\left.\phi\right|_{\tau_{2}}-\left.\phi\right|_{\tau_{1}}$ is the difference of $\phi$ at the ends $\tau_{2}$ and $\tau_{1}$ and is independent of $\tau \in\left[\tau_{1}, \tau_{2}\right]$. Likewise, the fourth integral can be expressed as $I_{4}=\int[\psi] s \mathrm{~d}^{3} \boldsymbol{a}$, because the entropy $s$ is independent of $\tau$ according to (31). This means that the gauge potentials $\phi$ and $\psi$ do not appear in the variations of the action $I$ for $\tau \in\left[\tau_{1}, \tau_{2}\right]$. In other words, the gauge potentials $\phi$ and $\psi$ make their appearance only in the action 
represented with the physical space coordinates $\boldsymbol{x}$. The fact that the last two Lagrangians in (39) do not contribute to the variations (for $\tau \in\left[\tau_{1}, \tau_{2}\right]$ ) implies that local Galilean symmetry is in fact an internal symmetry.

Thus, by omitting the integrated terms, we have

$$
I=\int L\left(X_{\mu}^{k}\right) \mathrm{d}^{4} a, \quad L=\frac{1}{2} X_{0}^{k} X_{0}^{k}-\epsilon\left(X_{l}^{k}, a^{k}\right),
$$

where $X_{l}^{k}=\partial X^{k} / \partial a^{l}, \quad X_{0}^{k}=X_{\tau}^{k}=v^{k} \quad(k, l=1,2,3)$, and $\mathrm{d}^{4} a=\mathrm{d} \tau \mathrm{d}^{3} \boldsymbol{a}$. In the $\boldsymbol{a}$-space, the point $\boldsymbol{a}$ is motion-less, while in the $\boldsymbol{x}$-space, there is a flow of particles driven by the potentials $\phi$ and $\psi$ according to (25).

\subsection{Noether's conserved currents}

The Euler-Lagrange equation can be writtten as

$$
\frac{\partial}{\partial a^{\mu}}\left(\frac{\partial L}{\partial X_{\mu}^{k}}\right)-\frac{\partial L}{\partial X^{k}}=\partial_{\mu}\left(\frac{\partial L}{\partial X_{\mu}^{k}}\right)-\frac{\partial L}{\partial X^{k}}=0
$$

$\left(\partial_{\mu}=\partial / \partial a^{\mu}\right)$. The energy-momentum tensor $T_{\mu}^{\nu}$ is defined by

$$
T_{\mu}^{\nu} \equiv X_{\mu}^{k}\left(\frac{\partial L}{\partial X_{\nu}^{k}}\right)-L \delta_{\mu}^{\nu}
$$

where $k=1,2,3$. As long as (41) is valid together with $\partial_{\tau} L=0$, we have a conservation equation $\partial_{\nu} T_{\mu}^{\nu}=0$. In fact, we have

$$
\begin{aligned}
\partial_{\nu} T_{\mu}^{\nu} & =\partial_{\nu}\left(X_{\mu}^{k}\right)\left(\frac{\partial L}{\partial X_{\nu}^{k}}\right)+X_{\mu}^{k} \partial_{\nu}\left(\frac{\partial L}{\partial X_{\nu}^{k}}\right)-\partial_{\mu} X^{k} \frac{\partial L}{\partial X^{k}}-\partial_{\mu}\left(X_{\nu}^{k}\right) \frac{\partial L}{\partial X_{\nu}^{k}} \\
& =X_{\mu}^{k}\left[\partial_{\nu}\left(\frac{\partial L}{\partial X_{\nu}^{k}}\right)-\frac{\partial L}{\partial X^{k}}\right]=0
\end{aligned}
$$

This is the Noether theorem (Noether (1918), Weinberg (1995)).

By using the Lagrangian $L$ of (40), we obtain $\partial L / \partial X_{0}^{k}=X_{0}^{k}=v^{k}$. The internal energy $\epsilon$ depends on the density $\rho$ which in turn depends on $X_{l}^{k}=$ $\partial X^{k} / \partial a^{l}$ by (38), where the entropy $s$ depends on $a^{l}$ only. Hence, we have

$$
\frac{\partial L}{\partial X_{l}^{k}}=-\frac{\partial \epsilon}{\partial \rho} \frac{\partial \rho}{\partial X_{l}^{k}}=-\frac{p}{\rho^{2}} \frac{\partial \rho}{\partial X_{l}^{k}},
$$


by using the thermodynamic relation given in (23). In view of (38), we obtain

$$
\frac{\partial L}{\partial X_{1}^{1}}=p \frac{\partial\left(X^{2}, X^{3}\right)}{\partial\left(a^{2}, a^{3}\right)}, \quad \frac{\partial L}{\partial X_{1}^{2}}=p \frac{\partial\left(X^{3}, X^{1}\right)}{\partial\left(a^{2}, a^{3}\right)}, \quad \frac{\partial L}{\partial X_{1}^{3}}=p \frac{\partial\left(X^{1}, X^{2}\right)}{\partial\left(a^{2}, a^{3}\right)} .
$$

Thus, the energy-momentum tensor $T_{\mu}^{\nu}$ are found as follows (Eckart 1960):

$$
\begin{aligned}
& T_{0}^{0}=X_{\tau}^{k}\left(\frac{\partial L}{\partial X_{\tau}^{k}}\right)-L=X_{\tau}^{k} X_{\tau}^{k}-L=\frac{1}{2} v^{2}+\epsilon \equiv H \text { (energy density), } \\
& T_{0}^{1}=X_{\tau}^{k}\left(\frac{\partial L}{\partial X_{1}^{k}}\right)=p \frac{\partial(X, Y, Z)}{\partial(\tau, b, c)}, \quad\left(\partial \epsilon / \partial \rho=p / \rho^{2}\right), \\
& T_{0}^{2}=p \frac{\partial(X, Y, Z)}{\partial(a, \tau, c)}, \quad T_{0}^{3}=p \frac{\partial(X, Y, Z)}{\partial(a, b, \tau)}
\end{aligned}
$$

Introducing the symbols $(\alpha, \beta, \gamma)$ to denote $(a, b, c)$ cyclically, another twelve components are

$$
\begin{aligned}
& T_{\alpha}^{0}=X_{\alpha}^{k}\left(\frac{\partial L}{\partial X_{0}^{k}}\right)=X_{\alpha}^{k} X_{\tau}^{k}=X_{\alpha} X_{\tau}+Y_{\alpha} Y_{\tau}+Z_{\alpha} Z_{\tau}, \equiv V_{\alpha}, \\
& T_{\alpha}^{\alpha}=X_{\alpha}^{k}\left(\frac{\partial L}{\partial X_{\alpha}^{k}}\right)-L=p \frac{\partial(X, Y, Z)}{\partial(\alpha, \beta, \gamma)}-\left(\frac{1}{2} v^{2}-\epsilon\right)=-\frac{1}{2} v^{2}+h, \\
& T_{\alpha}^{\beta}=X_{\alpha}^{k}\left(\frac{\partial L}{\partial X_{\beta}^{k}}\right)=0, \quad T_{\alpha}^{\gamma}=0 .
\end{aligned}
$$

The Noether conservation law $\partial_{\nu} T_{\mu}^{\nu}=0$ reduces to the momentum equations (see below) for $\mu=\alpha$ :

$$
\partial_{\tau} V_{\alpha}+\partial_{\alpha} F=0 \quad(\text { for } \alpha=a, b, c),
$$

where $F=-\frac{1}{2} v^{2}+h$, and other two equations are obtained with cyclic permutation of $(a, b, c)$. Integrating this with respect to $\tau$ between the limits 0 and $t$, we find the Weber's transformation (Lamb 1932, Art.15):

$$
V_{\alpha}(\tau)=X_{\alpha} X_{\tau}+Y_{\alpha} Y_{\tau}+Z_{\alpha} Z_{\tau}=V_{\alpha}(0)-\partial_{\alpha} \chi
$$

where $\chi=\int_{0}^{t} F \mathrm{~d} \tau=\int_{0}^{t}\left(-\frac{1}{2} v^{2}+h\right) \mathrm{d} \tau$. For $\mu=0$, the conservation law $\partial_{\nu} T_{\mu}^{\nu}=0$ describes the energy equation:

$$
\partial_{\tau} H+\partial_{a}\left[p \frac{\partial(X, Y, Z)}{\partial(\tau, b, c)}\right]+\partial_{b}\left[p \frac{\partial(X, Y, Z)}{\partial(a, \tau, c)}\right]+\partial_{c}\left[p \frac{\partial(X, Y, Z)}{\partial(a, b, \tau)}\right]=0 .
$$

In the Eulerian description with the independent variables $(t, x, y, z)$ instead of $(\tau, a, b, c)$, this reduces to

$$
\mathrm{D}_{t}\left(\frac{1}{2} v^{2}+h\right)=\frac{1}{\rho} \partial_{t} p
$$


This can be transformed to the following form of conservation of energy:

$$
\partial_{t}\left(\frac{1}{2} v^{2}+\epsilon\right)+\partial_{k}\left[\left(\frac{1}{2} v^{2}+h\right) v^{k}\right]=0 .
$$

Using (45) and (46), the equation (47) reduces to the equation for the acceleration $A_{\alpha}(\tau, \boldsymbol{a})$ :

$$
A_{\alpha} \equiv X_{\alpha} X_{\tau \tau}+Y_{\alpha} Y_{\tau \tau}+Z_{\alpha} Z_{\tau \tau}=-\frac{1}{\rho} \partial_{\alpha} p,
$$

which is known as the Lagrangian form of the equation of motion (Lamb 1932, Art.13). This is obtained by noting that $\partial_{\alpha} h=(1 / \rho) \partial_{\alpha} p$ and

$$
X_{\alpha \tau} X_{\tau}+Y_{\alpha \tau} Y_{\tau}+Z_{\alpha \tau} Z_{\tau}=\partial_{\alpha}\left(\frac{1}{2} v^{2}\right) .
$$

In view of (16), the equation (51) is equivalent to Euler's equation of motion in the Eulerian description of the barotropic relation $h(p)=\int^{p} \mathrm{~d} p^{\prime} / \rho\left(p^{\prime}\right)$ :

$$
\partial_{t} \boldsymbol{v}+(\boldsymbol{v} \cdot \nabla) \boldsymbol{v}=-\frac{1}{\rho} \nabla p=-\nabla h .
$$

Velocities $V_{\alpha}(\tau, \boldsymbol{a})$ in the $\boldsymbol{a}$-space may be determined by (48) with given initial conditions of $V_{\alpha}(0, \boldsymbol{a})$ and $h(0, \boldsymbol{a})$ at $\boldsymbol{a}=(a, b, c)=\boldsymbol{x}$. However, in order to transform it to the $\boldsymbol{x}$-space, there is some freedom. The equation (48) is invariant by rotational transformations of a displacement vector $\Delta \boldsymbol{X}=$ $(\Delta X, \Delta Y, \Delta Z)$ since the left hand side is of the form of a scalar product with respect to $\Delta \boldsymbol{X}$, so that the vector $\Delta \boldsymbol{X}$ is not uniquely determined (the density $\rho$ and enthalpy $h$ are not changed by this transformation since it is volumepreserving). The same freedom applies to the acceleration $A_{\alpha}(\tau, \boldsymbol{a})$ of $(51)$ as well. Rotational transformations are considered in the next section. This formulation helps to eliminate the arbitrariness.

\section{Rotational transformations}

Next, in order to equip the action $I$ with an additional mathematical structure of rotational symmetry (to be considered in $\S 6$ ), we summarize the rotational transformations here, and consider the rotational gauge symmetry. The related gauge group is the rotation group $S O(3)$. An infinitesimal rotation is described by the Lie algebra so(3) of three dimensions. The basis vectors of so(3) are denoted by $\left(e_{1}, e_{2}, e_{3}\right)$, which satisfy the commutation relations:

$$
\left[e_{j}, e_{k}\right]=\varepsilon_{j k l} e_{l},
$$


where $\varepsilon_{j k l}$ is the completely skew-symmetric third-order tensor, and

$$
e_{1}=\left[\begin{array}{ccc}
0 & 0 & 0 \\
0 & 0 & -1 \\
0 & 1 & 0
\end{array}\right], \quad e_{2}=\left[\begin{array}{ccc}
0 & 0 & 1 \\
0 & 0 & 0 \\
-1 & 0 & 0
\end{array}\right], \quad e_{3}=\left[\begin{array}{ccc}
0 & -1 & 0 \\
1 & 0 & 0 \\
0 & 0 & 0
\end{array}\right] \text {. }
$$

A rotation operator is defined by $\theta=\theta^{k} e_{k}$ where $\theta^{k}(k=1,2,3)$ are infinitesimal parameters:

$$
\theta=\left(\theta_{i j}\right) \equiv \theta^{k} e_{k}=\left[\begin{array}{ccc}
0 & -\theta^{3} & \theta^{2} \\
\theta^{3} & 0 & -\theta^{1} \\
-\theta^{2} & \theta^{1} & 0
\end{array}\right]
$$

Then, an infinitesimal rotation of the displacement vector $s=\left(s^{1}, s^{2}, s^{3}\right)$ is expressed by $\theta s$. This is also written as $\hat{\theta} \times \boldsymbol{s}$, where $\hat{\theta}=\left(\theta^{1}, \theta^{2}, \theta^{3}\right)$ is an infinitesimal angle vector.

Preliminary studies of the rotational symmetry of fluid flows are given in Kambe (2003a, b). However, more details are elaborated in the following subsections $5.1 \sim 5.3$, and the section 6 proposes a new Lagrangian $L_{A}$ to account for the rotation symmetry.

\subsection{Gauge transformation (Rotation symmetry)}

We consider local rotation of a fluid element about an arbitrary reference point $\boldsymbol{x}_{0}$ within a fluid. The non-abelian property of the rotational transformation requires infinitesimal analysis. At a neighboring point $\boldsymbol{x}_{0}+\boldsymbol{s}$ for a small $\boldsymbol{s}$, we consider its local transformation, $s \rightarrow s+\delta s$.

Rotational gauge transformation of a point $s$ in a frame $F$ (an inertial frame, say) to $s^{\prime}$ in a non-inertial frame $F^{\prime}$ is expressed as

$$
\boldsymbol{s}^{\prime}(\boldsymbol{s}, t)=\boldsymbol{s}+\delta \boldsymbol{s}(\boldsymbol{s}, t), \quad t^{\prime}=t,
$$

instead of (6) for the translation. The variation $\delta \boldsymbol{s}$ is defined by an infinitesimal rotation $\theta=\theta(t)$ (skew-symmetric) applied to the displacement vector $s$ : $\delta \boldsymbol{s} \equiv \boldsymbol{\eta}(\boldsymbol{s}, t)=\theta \boldsymbol{s}=\hat{\theta} \times \boldsymbol{s}$. The corresponding transformation of velocity $\left(\boldsymbol{v}=\mathrm{D}_{t} \boldsymbol{s}\right)$ is obtained from (55) as follows:

$$
\begin{aligned}
\boldsymbol{v}^{\prime}\left(\boldsymbol{s}^{\prime}\right)=\mathrm{D}_{t} \boldsymbol{s}^{\prime} & =\mathrm{D}_{t}(\boldsymbol{s}+\delta \boldsymbol{s})=\boldsymbol{v}(\boldsymbol{s})+\mathrm{D}_{t} \boldsymbol{\eta} \\
\delta \boldsymbol{v}(\boldsymbol{s}) & =\mathrm{D}_{t} \boldsymbol{\eta} \equiv \partial_{t} \boldsymbol{\eta}(\boldsymbol{s}, t)+\left(\boldsymbol{v} \cdot \nabla_{s}\right) \boldsymbol{\eta}(\boldsymbol{s}, t)=\partial_{t} \theta \boldsymbol{s}+\theta \boldsymbol{v}
\end{aligned}
$$


instead of (7) and (8) where $\nabla_{s}=\left(\partial / \partial s^{i}\right)$. As before (Sec. 2.3.2), in this transformation the points $\boldsymbol{s}$ and $\boldsymbol{s}^{\prime}$ are the same point with respect to the frame $F$. This means that the coordinate frame $F^{\prime}$ is rotated by an angle $-\hat{\theta}$ with respect to $F$ with a fixed origin $\boldsymbol{x}_{0}$. At the origin $\boldsymbol{x}_{0}(\boldsymbol{s}=0)$, we have the following:

$$
\delta \boldsymbol{v}(0)=\theta v=\theta^{k} e_{k} v,
$$

from (56) and (57), where $v=\boldsymbol{v}\left(\boldsymbol{x}_{0}\right)$.

\subsection{Acceleration}

Let us consider the time-derivative $\partial_{t} \boldsymbol{v}$ in the Euler equation (35) for a homentropic fluid. It is proposed that this term be replaced with $\mathrm{d}_{t} v$ as

$$
\partial_{t} v \rightarrow \mathrm{d}_{t} v=\partial_{t} v+\Omega v, \quad \Omega v=\Omega^{k} e_{k} v=\hat{\Omega} \times v
$$

where $\Omega$ is an operator defined by $\Omega=\Omega^{k} e_{k}$ for scalar fields $\Omega^{k}$, and $\hat{\Omega}$ is an axial vector defined by $\left(\Omega^{1}, \Omega^{2}, \Omega^{3}\right)$ The tensor $\Omega_{i j} \equiv\left(\Omega^{k} e_{k}\right)_{i j}$ is skewsymmetric $\left(\Omega_{i j}=-\Omega_{j i}\right)$.

We consider variations at the origin $(s=0)$. Then, we have $\delta v=\theta^{k} e_{k} v$ from (58). Variation of $\Omega^{k}$ is defined by 7

$$
\delta \Omega^{k}=\partial_{t} \theta^{k}+\varepsilon_{k l m} \theta^{l} \Omega^{m}
$$

Using $\delta \partial_{t}=\partial_{t^{\prime}}-\partial_{t}=-\partial_{t} \eta^{k} T_{k}$ from (9), we obtain the variation of $\mathrm{d}_{t} v$ :

$$
\begin{aligned}
\delta\left(\mathrm{d}_{t} v\right)=\partial_{t}(\delta v)+ & \left(\delta \partial_{t}\right) v+\delta \Omega^{k} e_{k} v+\Omega^{k} e_{k} \delta v=\partial_{t}\left(\theta^{k} e_{k} v\right)-\partial_{t}(\theta s)^{k} T_{k} v \\
& +\left(\partial_{t} \theta^{k}+\varepsilon_{k l m} \theta^{l} \Omega^{m}\right) e_{k} v+\Omega^{m} e_{m} \theta^{l} e_{l} v
\end{aligned}
$$

where $\varepsilon_{k l m}$ can be eliminated by using the following equality obtained from (53): $\varepsilon_{l m k} e_{k}=e_{l} e_{m}-e_{m} e_{l}$. Setting $s=0$, we obtain

$$
\delta\left(\mathrm{d}_{t} v\right)=\theta^{k} e_{k}\left[\partial_{t} v+\Omega^{m} e_{m} v\right]+2 \partial_{t} \theta^{k} e_{k} v=\hat{\theta} \times \mathrm{d}_{t} v+2 \partial_{t} \hat{\theta} \times v .
$$

The second term of the last expression denotes the Coriolis term in the rotating system. Thus, it is found that the covariant derivative $\mathrm{d}_{t} v$ behaves like the acceleration in a rotating frame, as far as $\Omega$ transforms according to (60).

In the previous analysis for the translation symmetry, it was shown that the left hand side of $(35), \partial_{t} \boldsymbol{v}+\nabla\left(\frac{1}{2} v^{2}\right)$, is in fact equal to the particle acceleration $\mathrm{D}_{t} \boldsymbol{v}$ of (36). In the present section taking into account the rotation symmetry,

${ }_{6}$ A simple font $v$ is used here for brevity instead of bold-face three-vector $\boldsymbol{v}$.

7 The vector form is $\delta \hat{\Omega}=\partial_{t} \hat{\theta}+\hat{\theta} \times \hat{\Omega}$. 
the partial derivative $\partial_{t}$ is replaced with the covariant derivative $\mathrm{d}_{t}=\partial_{t}+\Omega$. Thus, the particle acceleration $\nabla_{t} \boldsymbol{v}$ should be defined by

$$
\nabla_{t} \boldsymbol{v} \equiv \partial_{t} \boldsymbol{v}+\Omega \boldsymbol{v}+\operatorname{grad}\left(v^{2} / 2\right)=\partial_{t} \boldsymbol{v}+\hat{\Omega} \times \boldsymbol{v}+\operatorname{grad}\left(v^{2} / 2\right),
$$

where $\hat{\Omega}$ is the gauge field with respect to the rotation symmetry.

\subsection{Vorticity as a gauge field}

It can be verified that the gauge field $\hat{\Omega}$ in fact coincides with the vorticity $\nabla \times \boldsymbol{v}$ by the requirement of Galilean invariance of the covariant derivative $\nabla_{t} \boldsymbol{v}$ of (62), as follows. Under a global Galilean transformation from one frame $F: x=(t, \boldsymbol{x})$ to another $F_{*}: x_{*}=\left(t_{*}, \boldsymbol{x}_{*}\right)$ which is moving with a uniform velocity $\boldsymbol{U}$ relative to $F$, the position vector $\boldsymbol{x}_{*}$ and velocity vector $\boldsymbol{v}_{*}$ in the frame $F_{*}$ are given by $\boldsymbol{x}_{*}=\boldsymbol{x}-\boldsymbol{U} t$ and $\boldsymbol{v}_{*}=\boldsymbol{v}-\boldsymbol{U}$. Transformation laws of derivatives are $\partial_{t}=\partial_{t_{*}}-\boldsymbol{U} \cdot \boldsymbol{\nabla}_{*}$ and $\boldsymbol{\nabla}=\boldsymbol{\nabla}_{*}$. Applying these and replacing $\boldsymbol{v}$ by $\boldsymbol{v}_{*}+\boldsymbol{U}$, the covariant derivative of (62) is transformed to

$$
\begin{aligned}
& \left(\partial_{t_{*}}-\boldsymbol{U} \cdot \nabla_{*}\right)\left(\boldsymbol{v}_{*}+\boldsymbol{U}\right)+\hat{\Omega} \times\left(\boldsymbol{v}_{*}+\boldsymbol{U}\right)+\nabla_{*} \frac{1}{2}\left|\boldsymbol{v}_{*}+\boldsymbol{U}\right|^{2} \\
= & \partial_{t_{*}} \boldsymbol{v}_{*}+\hat{\Omega} \times \boldsymbol{v}_{*}+\nabla_{*}\left(v_{*}^{2} / 2\right)-\left(\boldsymbol{U} \cdot \nabla_{*}\right) \boldsymbol{v}_{*}+\hat{\Omega} \times \boldsymbol{U}+\nabla_{*}\left(\boldsymbol{v}_{*} \cdot \boldsymbol{U}\right),
\end{aligned}
$$

since $\boldsymbol{U}$ is a constant vector and $\nabla_{*}\left(U^{2}\right)=0$. We require the covariance, i.e. $\nabla_{t} \boldsymbol{v}=\left(\nabla_{t} \boldsymbol{v}\right)_{*}$. Namely, the right hand side should be equal to $\partial_{t_{*}} \boldsymbol{v}_{*}+\hat{\Omega}_{*} \times$ $\boldsymbol{v}_{*}+\nabla_{*}\left(v_{*}^{2} / 2\right)$. Therefore,

$$
\begin{aligned}
0 & =\left(\hat{\Omega}-\hat{\Omega}_{*}\right) \times \boldsymbol{v}_{*}-\left(\boldsymbol{U} \cdot \nabla_{*}\right) \boldsymbol{v}_{*}+\hat{\Omega} \times \boldsymbol{U}+\nabla_{*}\left(\boldsymbol{v}_{*} \cdot \boldsymbol{U}\right) \\
& =\left(\hat{\Omega}-\hat{\Omega}_{*}\right) \times \boldsymbol{v}_{*}+\left(\hat{\Omega}-\nabla_{*} \times \boldsymbol{v}_{*}\right) \times \boldsymbol{U}
\end{aligned}
$$

on using the following vector identity: $\boldsymbol{U} \times\left(\nabla_{*} \times \boldsymbol{v}_{*}\right)=-\left(\boldsymbol{U} \cdot \nabla_{*}\right) \boldsymbol{v}_{*}+\nabla_{*}\left(\boldsymbol{U} \cdot \boldsymbol{v}_{*}\right)$, for the constant vector $\boldsymbol{U}$. Equation (63) is satisfied identically, if

$$
\hat{\Omega}=\hat{\Omega}_{*}, \quad \hat{\Omega}=\nabla \times \boldsymbol{v}=\nabla_{*} \times \boldsymbol{v}_{*}=\hat{\Omega}_{*} .
$$

The second relation holds by the Galilean transformation since $\nabla=\nabla_{*}$ and $\boldsymbol{v}=\boldsymbol{v}_{*}+\boldsymbol{U}$. Thus, the Galilean invariance of $\nabla_{t} \boldsymbol{v}$ results in

$$
\hat{\Omega}=\nabla \times \boldsymbol{v} \equiv \boldsymbol{\omega}
$$

Namely, the gauge field $\hat{\Omega}$ coincides with the vorticity $\boldsymbol{\omega}$. Consequently, the covariant derivative $\nabla_{t} \boldsymbol{v}$ (particle acceleration) is given by

$$
\nabla_{t} \boldsymbol{v}=\partial_{t} \boldsymbol{v}+\nabla\left(\frac{1}{2} v^{2}\right)+\boldsymbol{\omega} \times \boldsymbol{v}
$$


Using the vector identity

$$
\boldsymbol{v} \times(\nabla \times \boldsymbol{v})=\nabla\left(\frac{1}{2}|\boldsymbol{v}|^{2}\right)-(\boldsymbol{v} \cdot \nabla) \boldsymbol{v}
$$

the last expression (66) is transformed to the convective derivative of $\boldsymbol{v}$ (i.e. the particle acceleration):

$$
\nabla_{t} \boldsymbol{v}=\partial_{t} \boldsymbol{v}+(\boldsymbol{v} \cdot \nabla) \boldsymbol{v}
$$

Thus, we have $\nabla_{t}=\partial_{t}+(\boldsymbol{v} \cdot \nabla)$. So far, $\boldsymbol{x}_{0}+\boldsymbol{s}$ was a local position vector around a fixed point $\boldsymbol{x}_{0}$. In general, using $\boldsymbol{x}$ in place of $\boldsymbol{x}_{0}+\boldsymbol{s}$, we arrive at the same definition: $\boldsymbol{v}(\boldsymbol{x}, t)=\nabla_{t} \boldsymbol{x}=\left(\partial_{t}+(\boldsymbol{v} \cdot \nabla)\right) \boldsymbol{x}$.

\section{Lagrangian $L_{A}$ associated with rotation symmetry}

Associated with the rotation symmetry, we try to introduce an additional Lagrangian according to the gauge principle. The property of $I_{3}$ and $I_{4}$ considered below the equation (39) suggests how to find such a Lagrangian. It was seen there that the Lagrangians represented with the particle coordinates $\boldsymbol{a}=\left(a^{1}, a^{2}, a^{3}\right)=(a, b, c)$ can be integrated with respect to the time $\tau$ and eliminated in the action to deduce the Euler-Lagrange equation, while they are non-trivial in the physical-space coordinates $\boldsymbol{x}=\left(x^{1}, x^{2}, x^{3}\right)=(x, y, z)$ because of the non-trivial Jacobian matrix $\left(\partial x^{k} / \partial a^{l}\right)$ of the transformation. As a result, the action principle yields the continuity equation and entropy equation. Thus the gauge potentials are regarded as mathematical agents with mechanical functions that drive fluid motions in the physical $\boldsymbol{x}$-space, whereas they disappear in motionless $\boldsymbol{a}$-space.

It is important to observe that, in the Lagrangian (39), the integrands of the last two integarals are of the form $\partial_{\tau}(\cdot)$, because the entropy $s$ and mass element $\rho \mathrm{d}^{3} \boldsymbol{x}=\mathrm{d}^{3} \boldsymbol{a}$ are independent of $\tau$. The action is defined by $I=$ $\iint\left[L^{\prime}+\partial_{\tau}(\cdot)\right] \mathrm{d} \tau \mathrm{d}^{3} \boldsymbol{a}$, where $L^{\prime}$ denotes the part of the first two terms of (39). This property is the simplest case of representation of topology in the gauge theory. In the context of rotational flows, it is known that the helicity (or Hopf invariant, Arnold \& Khesin (1998)) defined by (34) describes non-trivial topology of vorticity field, i.e. mutual linking of vorticity lines. This is closely related with the Chern-Simons term in the gauge theory. The Chern-Simons term lives in one dimension lower than the original four-space-time $\left(x^{\mu}\right)$ of the action $I$ because a topological term in the action is expressed in a form of total divergence $\left(\partial_{\mu} F^{\mu}\right)$ and characterizes topologically non-trivial structures of the gauge field (Chern 1979; Jackiw 1985; Desser et al. 1982). Here, we follow the formulation of Sec.4.1 and look for a $\tau$-independent field directly. 


\subsection{Lagrangian in $\boldsymbol{a}$-space}

The $\tau$-independent field can be found immediately from Eq. (47). Taking the curl of this equation with respect to the coordinates $(a, b, c)$, we obtain

$$
\nabla_{a} \times \partial_{\tau} \boldsymbol{V}_{a}=\partial_{\tau}\left(\nabla_{a} \times \boldsymbol{V}_{a}\right)=0
$$

since $\nabla_{a} \times \nabla_{a} F=0$, where $\nabla_{a}=\left(\partial_{a}, \partial_{b}, \partial_{c}\right)$, and $\boldsymbol{V}_{a}=\left(V_{a}, V_{b}, V_{c}\right)$ is defined by (45). It is found that $\nabla_{a} \times \boldsymbol{V}_{a}$ is independent of $\tau 8$ Hence, one may write as $\nabla_{a} \times \boldsymbol{V}_{a}=\boldsymbol{\Omega}_{a}(\boldsymbol{a})$, where the right hand side is a vector depending on $\boldsymbol{a}$ only. It is useful to formulate this property in the framework of exterior differential forms and Lie derivative. Vector $\boldsymbol{V}_{a}$ is understood as a transformed form of the velocity $\boldsymbol{v}=\left(X_{\tau}, Y_{\tau}, Z_{\tau}\right)=(u, v, w)$ into the $\boldsymbol{a}$-space. This is seen on the basis of a 1 -form $\mathcal{V}$ defined by

$$
\begin{aligned}
\mathcal{V} & =V_{a} \mathrm{~d} a+V_{b} \mathrm{~d} b+V_{c} \mathrm{~d} c \quad\left(\text { written as } \boldsymbol{V}_{a} \cdot \mathrm{d} \boldsymbol{a}\right) \\
& =u \mathrm{~d} x+v \mathrm{~d} y+w \mathrm{~d} z .
\end{aligned}
$$

where $V_{a}=u x_{a}+v y_{a}+w z_{a}, x_{a}=\partial X / \partial a, u=X_{\tau}$, etc. and $\mathrm{d} \boldsymbol{a}=(\mathrm{d} a, \mathrm{~d} b, \mathrm{~d} c)$. Hence $V_{a}$ is equivalent to (45). Its differential $\mathrm{d} \mathcal{V}$ gives a two-form $\Omega^{2}=\mathrm{d} \mathcal{V}$ :

$$
\begin{aligned}
\Omega^{2}=\mathrm{d} \mathcal{V} & =\Omega_{a} \mathrm{~d} b \wedge \mathrm{d} c+\Omega_{b} \mathrm{~d} c \wedge \mathrm{d} a+\Omega_{c} \mathrm{~d} a \wedge \mathrm{d} b=\boldsymbol{\Omega}_{a} \cdot \boldsymbol{S}^{2} \\
& =\omega_{x} \mathrm{~d} y \wedge \mathrm{d} z+\omega_{y} \mathrm{~d} z \wedge \mathrm{d} x+\omega_{z} \mathrm{~d} x \wedge \mathrm{d} y=\boldsymbol{\omega} \cdot \boldsymbol{s}^{2}
\end{aligned}
$$

where $\nabla_{a} \times \boldsymbol{V}_{a}=\left(\Omega_{a}, \Omega_{b}, \Omega_{c}\right)=\Omega_{a}$, and $\nabla \times \boldsymbol{v}=\left(\omega_{x}, \omega_{y}, \omega_{z}\right)=\boldsymbol{\omega}$ is the vorticity. Thus, it is seen that $\Omega_{a}$ is the vorticity transformed to the $\boldsymbol{a}$-space, and the 2-forms $\boldsymbol{s}^{2}$ and $\boldsymbol{S}^{2}$ are surface forms defined in the footnot 9 . The equation (69) is transformed to the $\tau$-derivative of the 2 -form $\Omega^{2}$, which is understood as the Lie derivative: $\mathcal{L}_{\partial_{\tau}} \Omega^{2}=0$. Its representation in the $(\boldsymbol{x}, t)$ space is given as follows.

It is useful to define two tangent vectors $W$ and $V$ (tangent to the flow generated by $\boldsymbol{v})$ :

$$
W \equiv \partial_{t}+V, \quad V \equiv u \partial_{x}+v \partial_{y}+w \partial_{z}=v^{k} \partial_{k}=\boldsymbol{v} \cdot \nabla
$$

where $V$ is regarded as a tangent vector dual to the 1 -form $\mathcal{V}$ of $(71)$. The Lie derivative $\mathcal{L}_{\partial_{\tau}}$ can be defined by

$$
\mathcal{L}_{\partial_{\tau}}=\mathcal{L}_{W}=\mathcal{L}_{\partial_{t}+V}=\partial_{t}+\mathcal{L}_{V}
$$

$\overline{8}$ This property can be related to the invariance of the Lagrangian with respect to the relabeling transformation $\boldsymbol{a} \rightarrow \boldsymbol{a}^{\prime}$, satisfying the mass invariance $\mathrm{d}^{3} \boldsymbol{a}^{\prime}=\mathrm{d}^{3} \boldsymbol{a}$.

${ }^{9} \boldsymbol{s}^{2}=\left(\mathrm{d} x^{2} \wedge \mathrm{d} x^{3}, \mathrm{~d} x^{3} \wedge \mathrm{d} x^{1}, \mathrm{~d} x^{1} \wedge \mathrm{d} x^{2}\right)$, and $\boldsymbol{S}^{2}=\left(\mathrm{d} a^{2} \wedge \mathrm{d} a^{3}, \mathrm{~d} a^{3} \wedge \mathrm{d} a^{1}, \mathrm{~d} a^{1} \wedge \mathrm{d} a^{2}\right)$. 
according to the symbols of differential calculus and tangent vector, where $V=v^{k} \partial_{k}$ is also a differential operator along a streamline generated by the flow $(u, v, w)$. By applying the Lie derivative $\mathcal{L}_{\partial_{\tau}}$ on the 2-form $\Omega^{2}$ (Frankel 1997, Ch.4.2), we obtain

$$
\begin{aligned}
0=\mathcal{L}_{\partial_{\tau}} \Omega^{2} & =\partial_{t} \Omega^{2}+\mathcal{L}_{V} \Omega^{2} \\
& =\partial_{t} \boldsymbol{\omega} \cdot \boldsymbol{s}^{2}+[\boldsymbol{v}, \boldsymbol{\omega}] \cdot \boldsymbol{s}^{2}+(\nabla \cdot \boldsymbol{v}) \boldsymbol{\omega} \cdot \boldsymbol{s}^{2} \\
& =E_{W}[\boldsymbol{\omega}] \cdot \boldsymbol{s}^{2}
\end{aligned}
$$

where $[\boldsymbol{v}, \boldsymbol{\omega}]$ is the Lie bracket defined by $(\boldsymbol{v} \cdot \nabla) \boldsymbol{\omega}-(\boldsymbol{\omega} \cdot \nabla) \boldsymbol{v}$, and

$$
E_{W}[\boldsymbol{\omega}] \equiv \partial_{t} \boldsymbol{\omega}+[\boldsymbol{v}, \boldsymbol{\omega}]+(\nabla \cdot \boldsymbol{v}) \boldsymbol{\omega}=\partial_{t} \boldsymbol{\omega}+\nabla \times(\boldsymbol{\omega} \times \boldsymbol{v}),
$$

where $\nabla \cdot \boldsymbol{\omega}=0$ is used. Thus, we obtain the vorticity equation from (75):

$$
\partial_{t} \boldsymbol{\omega}+\nabla \times(\boldsymbol{\omega} \times \boldsymbol{v})=0
$$

Next, let us introduce a gauge vector-potential $\boldsymbol{A}_{a}=\left(A_{a}, A_{b}, A_{c}\right)$ in the $\boldsymbol{a}$ space, and define its 1 -form $A^{1}$ by

$$
\begin{aligned}
A^{1} & =A_{a} \mathrm{~d} a+A_{b} \mathrm{~d} b+A_{c} \mathrm{~d} c \quad\left(\text { written as } \boldsymbol{A}_{a} \cdot \mathrm{d} \boldsymbol{a}\right) \\
& =A_{x} \mathrm{~d} x+A_{y} \mathrm{~d} y+A_{z} \mathrm{~d} z
\end{aligned}
$$

The exterior product of $A^{1}$ and $\Omega^{2}$ results in a volume form $\mathrm{d}^{3} \boldsymbol{a}=\mathrm{d} a \wedge(\mathrm{d} b \wedge$ $\mathrm{d} c)=\mathrm{d} b \wedge(\mathrm{d} c \wedge \mathrm{d} a)=\mathrm{d} c \wedge(\mathrm{d} a \wedge \mathrm{d} b)$ multiplied by a scalar $\left\langle\boldsymbol{A}_{a}, \boldsymbol{\Omega}_{a}\right\rangle$ :

$$
A^{1} \wedge \Omega^{2}=\left(\boldsymbol{A}_{a} \cdot \mathrm{d} \boldsymbol{a}\right) \wedge\left(\boldsymbol{\Omega}_{a} \cdot \boldsymbol{S}^{2}\right)=\left\langle\boldsymbol{A}_{a}, \boldsymbol{\Omega}_{a}\right\rangle \mathrm{d}^{3} \boldsymbol{a}
$$

where $\left\langle\boldsymbol{A}_{a}, \boldsymbol{\Omega}_{a}\right\rangle=A_{a} \Omega_{a}+A_{b} \Omega_{b}+A_{c} \Omega_{c}$ is a scalar product of $\boldsymbol{A}_{a}$ and $\boldsymbol{\Omega}_{a}$.

In the $\boldsymbol{x}=(x, y, z)$ space, the same exterior product is given by

$$
A^{1} \wedge \Omega^{2}=\langle\boldsymbol{A}, \boldsymbol{\omega}\rangle \mathrm{d}^{3} \boldsymbol{x}, \quad \mathrm{d}^{3} \boldsymbol{x}=\mathrm{d} x \wedge \mathrm{d} y \wedge \mathrm{d} z .
$$

It is obvious that the scalar product $\langle\boldsymbol{A}, \boldsymbol{\omega}\rangle$ is invariant under local rotational transformations in the $\boldsymbol{x}$ space.

Thus, it is proposed that a possible type of Lagrangian is of the form,

$$
\Lambda_{A}=-\int_{M}\left\langle\partial_{\tau} \boldsymbol{A}_{a}, \boldsymbol{\Omega}_{a}\right\rangle \mathrm{d}^{3} \boldsymbol{a}=-\int_{M} \mathcal{L}_{\partial_{\tau}}\left[\left\langle\boldsymbol{A}_{a}, \boldsymbol{\Omega}_{a}\right\rangle \mathrm{d}^{3} \boldsymbol{a}\right],
$$

where the time derivative $\partial_{\tau}$ was taken out of the inner-product since $\Omega_{a}$ is independent of $\tau$. It follows that $\Lambda_{A}$ can be dropped in the action $I$ expressed with the coordinate $\boldsymbol{a}$ except the integrated terms depending on the initial and final times of $\tau$-integration. However, this Lagrangian becomes non-trivial when transformed to the physical-space coordinate $\boldsymbol{x}$ because of the non-trivial Jacobian of transformation. It is shown later that $\boldsymbol{A}_{a}$ may be understood as a kind of vector potential associated with the gauge field $\boldsymbol{\Omega}_{a}$. 


\subsection{Eulerian coordinates}

From the equality of (79) and (80), we have $\left\langle\boldsymbol{A}_{a}, \boldsymbol{\Omega}_{a}\right\rangle \mathrm{d}^{3} \boldsymbol{a}=\langle\boldsymbol{A}, \boldsymbol{\omega}\rangle \mathrm{d}^{3} \boldsymbol{x}$. Using the definition (74),

$$
\mathcal{L}_{\partial_{\tau}}\left[\left\langle\boldsymbol{A}_{a}, \boldsymbol{\Omega}_{a}\right\rangle \mathrm{d}^{3} \boldsymbol{a}\right]=\mathcal{L}_{W}\left[A^{1} \wedge \Omega^{2}\right]=\left[\mathcal{L}_{W} A^{1}\right] \wedge \Omega^{2}
$$

where (75) was used. With the same calculus as in (80), the right hand side is given by $\left[\mathcal{L}_{W} A^{1}\right] \wedge \Omega^{2}=\left\langle\mathcal{L}_{W} \boldsymbol{A}, \boldsymbol{\omega}\right\rangle \mathrm{d}^{3} \boldsymbol{x}$, where the Lie derivative of a 1 -form $A^{1}$ is defined by

$$
\begin{aligned}
\mathcal{L}_{W} A^{1} & :=\left(\partial_{t} A_{i}+v^{k} \partial_{k} A_{i}+A_{k} \partial_{i} v^{k}\right) \mathrm{d} x^{i}=\Psi_{i} \mathrm{~d} x^{i}, \\
\Psi_{i} & :=\left(\mathcal{L}_{W} \boldsymbol{A}\right)_{i}=\partial_{t} A_{i}+v^{k} \partial_{k} A_{i}+A_{k} \partial_{i} v^{k}
\end{aligned}
$$

(e.g. Frankel (1997), §4.2c) 10 . Thus, the Lagrangian $\Lambda_{A}$ in the $\boldsymbol{x}$ variables is

$$
\Lambda_{A}=-\int_{M}\left\langle\mathcal{L}_{W} \boldsymbol{A}, \boldsymbol{\omega}\right\rangle \mathrm{d}^{3} \boldsymbol{x}=-\int_{M}\langle\boldsymbol{\Psi}, \boldsymbol{\omega}\rangle \mathrm{d}^{3} \boldsymbol{x}, \quad \boldsymbol{\Psi} \equiv \mathcal{L}_{W} \boldsymbol{A}
$$

Integrating this by parts and using the relation $\boldsymbol{\omega}=\nabla \times \boldsymbol{v}$, we have

$$
\Lambda_{A}=-\int_{M}\langle\nabla \times \Psi, \boldsymbol{v}\rangle \mathrm{d}^{3} \boldsymbol{x}+\operatorname{Int}_{S}=-\int_{M} \rho\langle\boldsymbol{b}, \boldsymbol{v}\rangle \mathrm{d}^{3} \boldsymbol{x}+\operatorname{Int}_{S} .
$$

where $\operatorname{Int}_{S}$ denotes the integral over the surface $S$ bounding $M$. This is the proposed Lagrangian $\Lambda_{A}$ in the physical space $\boldsymbol{x}$, where

$$
\rho \boldsymbol{b} \equiv \nabla \times \boldsymbol{\Psi}=\nabla \times\left(\mathcal{L}_{W} \boldsymbol{A}\right) .
$$

The Lagrangian $\Lambda_{A}$ of (83) is an integral of $\left\langle\mathcal{L}_{W} \boldsymbol{A}, \boldsymbol{\omega}\right\rangle \equiv \mathcal{L}_{W} A^{1}[\boldsymbol{\omega}]$. Given a vector field $Y$, the value $\mathcal{L}_{W} A^{1}[Y]$ taken on the vector $Y$ measures the derivative of $A^{1}$ (as one moves along the trajectory of $W$, i.e. the particle path) evaluated on a vector field $Y$ frozen to the flow generated by $W$.

Using (82) in (83) and carrying out integration by parts, we obtain

$$
\Lambda_{A}=\int_{M}\left\langle\boldsymbol{A}, E_{W}[\boldsymbol{\omega}]\right\rangle \mathrm{d}^{3} \boldsymbol{x}
$$

with $E_{W}[\boldsymbol{\omega}]$ defined in (76), where the integrated terms are dropped. Here, we redefine $\Lambda_{A}$ by this equation instead of (83). Hence, the expressions (83) and (84) must be modified by additional terms of surface integral over $S$, which are to be expressed symbolically with $\operatorname{Int}_{S}$. This Lagrangian (86) provides a source term of non-vanishing helicity. An example of such a flow will be given in the last section 8.3.

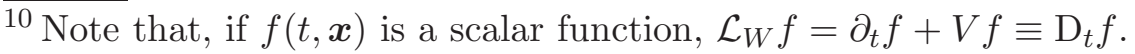




\section{Corrections to the previous Variation}

The Lagrangian $\Lambda_{A}$ of (84) should be included in the total Lagrangian to describe rotational flows of an ideal fluid. Accordingly, the Lagrangian density of the total Lagrangian (excluding surface terms) is defined by

$$
\begin{aligned}
L_{\mathrm{T}}=L_{\mathrm{T}}[\boldsymbol{v}, \rho, s, \phi, \psi, \boldsymbol{b}] & \equiv \frac{1}{2} \rho\langle\boldsymbol{v}, \boldsymbol{v}\rangle-\rho \epsilon(\rho, s)-\rho\left(\partial_{t}+\boldsymbol{v} \cdot \nabla\right) \phi \\
& -\rho s\left(\partial_{t}+\boldsymbol{v} \cdot \nabla\right) \psi-\rho\langle\boldsymbol{b}, \boldsymbol{v}\rangle .
\end{aligned}
$$

As before (Sec. 3.2), we take variations of the field variables $\boldsymbol{v}, \rho, s$ and potentials $\phi$ and $\psi$. In addition, we must now include the variation of the new variable $\boldsymbol{A}$. Independent variations are taken for those variables. Substituting the varied variables $\boldsymbol{v}+\delta \boldsymbol{v}, \rho+\delta \rho, s+\delta s, \phi+\delta \phi, \psi+\delta \psi$ and $\boldsymbol{A}+\delta \boldsymbol{A}$ into $L_{\mathrm{T}}[\boldsymbol{v}, \rho, s, \phi, \psi, \boldsymbol{A}]$ and writing its variation as $\delta L_{\mathrm{T}}$, we obtain

$$
\begin{aligned}
& \delta L_{\mathrm{T}}=\delta \boldsymbol{v} \cdot \rho(\boldsymbol{v}-\nabla \phi-s \nabla \psi-\boldsymbol{w})-\delta s \rho \mathrm{D}_{t} \psi \\
& \quad+\delta \rho\left(\frac{1}{2} u^{2}-h-\mathrm{D}_{t} \phi-s \mathrm{D}_{t} \psi-\boldsymbol{v} \cdot \boldsymbol{b}\right)+\left\langle\delta \boldsymbol{A}, E_{W} \boldsymbol{\omega}\right\rangle \\
& \quad+\quad \cdots \cdots
\end{aligned}
$$

where remaining terms denoted by $\cdots \cdots$ are omitted since they are the same as (24). The new term $\boldsymbol{w}$ is defined by

$$
\begin{aligned}
\frac{\delta \Lambda_{A}}{\delta v^{k}} \delta v^{k} & =-\rho\langle\boldsymbol{w}, \delta \boldsymbol{v}\rangle, \\
\boldsymbol{w} & =\left(w_{i}\right), \quad w_{i}=\left(\partial / \partial v^{i}\right)\langle\boldsymbol{v}, \boldsymbol{b}\rangle .
\end{aligned}
$$

Thus, the variational principle, $\delta I=0$ for independent variations of $\delta \boldsymbol{v}, \delta \rho$, $\delta \boldsymbol{A}$, etc. results in

$$
\begin{aligned}
\delta \boldsymbol{v}: & \boldsymbol{v}-\nabla \phi-s \nabla \psi-\boldsymbol{w}=0, \\
\delta \rho: & \frac{1}{2} v^{2}-h-\mathrm{D}_{t} \phi-s \mathrm{D}_{t} \psi-\boldsymbol{v} \cdot \boldsymbol{b}=0, \\
\delta \boldsymbol{A}: & E_{W} \boldsymbol{\omega}=0,
\end{aligned}
$$

where the omitted terms $\cdots \cdots$ are the same as before. Thus, the continuity equation (29) and entropy equation (30) are obtained as before. The equation (91) gives an expression for the velocity $\boldsymbol{v}$ :

$$
\boldsymbol{v}=\nabla \phi+s \nabla \psi+\boldsymbol{w} .
$$

The vorticity $\boldsymbol{\omega}=\nabla \times \boldsymbol{v}$ is represented by

$$
\boldsymbol{\omega}=\nabla s \times \nabla \psi+\nabla \times \boldsymbol{w} .
$$


The second term $\nabla \times \boldsymbol{w}$ is a new term leading to non-vanishing vorticity in a homentropic fluid of uniform $s$. The equation (93) is the same as (77) by (76).

Potential part and rotational part:

The potential part of the present problem is described by (92), and the rotational part by (77). In order to show that this is consisitent with the traditional formulation, suppose that the vorticity equation (93) is solved and a velocity field $\boldsymbol{v}(\boldsymbol{x}, t)$ is found. In view of the relation $\boldsymbol{\omega}=\nabla \times \boldsymbol{v}$, the velocity $\boldsymbol{v}$ should satisfy the following:

$$
\partial_{t} \boldsymbol{v}+\boldsymbol{\omega} \times \boldsymbol{v}=\nabla H^{\prime}
$$

for a scalar function $H^{\prime}(\boldsymbol{x}, t)$. Obviously, taking the curl of this equation reduces to (77).

On the other hand, according to Sec.4.2, we have the Euler's equation of motion (52), because the new Lagrangian $\Lambda_{A}$ has no effect in the total Lagrangian of $\boldsymbol{a}$ space owing to its construction, and hence generates no new term. Using the identity (67), this reduces to the following:

$$
\partial_{t} \boldsymbol{v}+\boldsymbol{\omega} \times \boldsymbol{v}=-\nabla\left(\frac{1}{2} v^{2}+h\right) .
$$

Defining $H^{\prime}$ by $H^{\prime}=\partial_{t} \phi+s \partial_{t} \psi+\boldsymbol{v} \cdot(\boldsymbol{b}-\boldsymbol{w})$, the equation (92) is

$$
\begin{aligned}
0= & \frac{1}{2} v^{2}-h-\mathrm{D}_{t} \phi-s \mathrm{D}_{t} \psi-\boldsymbol{v} \cdot \boldsymbol{b}=\frac{1}{2} v^{2}-h-\partial_{t} \phi \\
& -s \partial_{t} \psi-\boldsymbol{v} \cdot(\nabla \phi+s \nabla \psi+\boldsymbol{b})=-\frac{1}{2} v^{2}-h-H^{\prime}
\end{aligned}
$$

by using (94). In fact, it can be shown that both expressions (96) and (97) are equivalent with this $H^{\prime}$, as follows. First we note the following identities:

$$
\begin{aligned}
(\nabla \times \boldsymbol{w}) \times \boldsymbol{v} & =(\boldsymbol{v} \cdot \nabla) \boldsymbol{w}+w^{k} \nabla v^{k}-\nabla(\boldsymbol{v} \cdot \boldsymbol{w}), \\
w^{k} \nabla v^{k}-(\boldsymbol{v} \cdot \nabla) \nabla \phi & =\nabla\left(\frac{1}{2} v^{2}-(\boldsymbol{v} \cdot \nabla) \phi\right)-s \partial_{k} \psi \nabla v^{k}
\end{aligned}
$$

The second is an identity with $\boldsymbol{v}$ given by (94). Using these, one can verify the following:

$$
\partial_{t} \boldsymbol{w}+(\nabla \times \boldsymbol{w}) \times \boldsymbol{v}+\nabla[\boldsymbol{v} \cdot(\boldsymbol{w}-\boldsymbol{b})]=\partial_{t} \boldsymbol{v}+(\boldsymbol{v} \cdot \nabla) \boldsymbol{v}+\nabla h .
$$

The right hand side vanishes by Eq.(52). Therefore, we obtain

$$
\partial_{t} \boldsymbol{w}+(\nabla \times \boldsymbol{w}) \times \boldsymbol{v}=-\nabla[\boldsymbol{v} \cdot(\boldsymbol{w}-\boldsymbol{b})] .
$$

It is not difficult to show that the equation (96) reduces to this equation for the above $H^{\prime}$ and the total velocity $\boldsymbol{v}$ of (94), where the total vorticity $\boldsymbol{\omega}$ is given by (95). The equation (101) is a new equation to be satisfied by the rotational component $\boldsymbol{w}$. 
If $s=s_{0}$ (uniform), then $\boldsymbol{v}=\nabla \Phi+\boldsymbol{w}\left(\Phi=\phi+s_{0} \psi\right)$, and $\boldsymbol{\omega}=\nabla \times \boldsymbol{w}$. Subtracting (101) from (97), we obtain $\nabla\left[\frac{1}{2} v^{2}+h+\partial_{t} \Phi+\boldsymbol{v} \cdot(\boldsymbol{b}-\boldsymbol{w})\right]=0$, which is equivalent to (98).

Thus there is no contradiction between the traditional formulation and the present one. New aspects of the present formulation are described in the next section, in addition to the expression (101) for the rotational component $\boldsymbol{w}$.

\section{Significance of $\Lambda_{A}$}

We consider here the significance of the Lagrangian $\Lambda_{A}$ associated with rotation symmetry and its outcome derived from the variational principle.

\subsection{Source of helicity}

Let us recall that $\rho \boldsymbol{b}$ is defined by (85). Using (82), the expression of $\boldsymbol{\Psi}$ is rewritten as

$$
\boldsymbol{\Psi}=\partial_{t} A+v^{k} \partial_{k} A+A_{k} \nabla v^{k}=\partial_{t} A+(\nabla \times A) \times \boldsymbol{v}+\nabla\left(A_{k} v^{k}\right) .
$$

Therefore, defining a vector $\boldsymbol{B}$ with $\boldsymbol{B}=\nabla \times A$, we have

$$
\rho \boldsymbol{b}=\nabla \times \boldsymbol{\Psi}=\partial_{t} \boldsymbol{B}+\nabla \times(\boldsymbol{B} \times \boldsymbol{v})=E_{W}[\boldsymbol{B}] .
$$

Variation of $\Lambda_{A}$ of (84) with respect to a variation $\delta \boldsymbol{v}$ is given by

$$
\delta \Lambda_{A}=-\int_{M}[\langle\nabla \times \boldsymbol{\Psi}, \delta \boldsymbol{v}\rangle+\langle\nabla \times \delta \boldsymbol{\Psi}, \boldsymbol{v}\rangle] \mathrm{d}^{3} \boldsymbol{x},
$$

where $\nabla \times \delta \boldsymbol{\Psi}=\nabla \times(\boldsymbol{B} \times \delta \boldsymbol{v})$ from (102) for the variation $\delta \boldsymbol{v}$ on keeping $\boldsymbol{A}$ (hence $\boldsymbol{B}$ ) fixed. Therefore, we have

$$
\delta \Lambda_{A}=-\int_{M}\langle(\nabla \times \boldsymbol{\Psi}+\boldsymbol{\omega} \times \boldsymbol{B}), \delta \boldsymbol{v}\rangle \mathrm{d}^{3} \boldsymbol{x} .
$$

This leads to the following expression for $\boldsymbol{w}$ defined by (89):

$$
\rho \boldsymbol{w}=\nabla \times \boldsymbol{\Psi}+\boldsymbol{\omega} \times \boldsymbol{B}=E_{W}[\boldsymbol{B}]+\boldsymbol{\omega} \times \boldsymbol{B},
$$

where $E_{W}[\boldsymbol{B}] \equiv \partial_{t} \boldsymbol{B}+\nabla \times(\boldsymbol{B} \times \boldsymbol{v})$ from $(76)$.

For flows of a homentropic fluid with constant entropy $s_{0}$, the velocity is given by (94): $\boldsymbol{v}=\nabla \Phi+\boldsymbol{w}\left(\Phi=\phi+s_{0} \psi\right)$. Therefore, the helicity is

$$
H=\int_{V} \boldsymbol{\omega} \cdot \boldsymbol{v} \mathrm{d}^{3} \boldsymbol{x}=\int_{V} \boldsymbol{\omega} \cdot \boldsymbol{w} \mathrm{d}^{3} \boldsymbol{x}=\int_{V} \rho^{-1} \boldsymbol{\omega} \cdot E_{W}[\boldsymbol{B}] \mathrm{d}^{3} \boldsymbol{x},
$$


since $\nabla \cdot \boldsymbol{\omega}=0$. Thus it is found that the term $E_{W}[\boldsymbol{B}]$ generates the helicity.

\subsection{Uniqueness of transformation}

Transformation from the Lagrangian $\boldsymbol{a}$ space to Eulerian $\boldsymbol{x}(\boldsymbol{a})$ space is determined locally by nine components of the matrix $\partial x^{k} / \partial a^{l}$. However, in the previous solution considered in Sec.4.2, we had three relations (48) between the $\boldsymbol{x}$-velocity $\boldsymbol{v}=\left(X_{\tau}, Y_{\tau}, Z_{\tau}\right)$ and the $\boldsymbol{a}$-velocity $\left(V_{a}, V_{b}, V_{c}\right)$, and another three relations (51) between the $\boldsymbol{x}$-acceleration $\mathcal{A}=\left(X_{\tau \tau}, Y_{\tau \tau}, Z_{\tau \tau}\right)$ and the $\boldsymbol{a}$-acceleration $\left(A_{a}, A_{b}, A_{c}\right)$. These six relations are not sufficient to determine the nine matrix elements $\partial x^{k} / \partial a^{l}$. But now, we are equipped with a new structure of rotational symmetry which is expected to resolve this indefiniteness.

In fact, the remaining three conditions are found from the equation (72) connecting the $\boldsymbol{x}$-vorticity $\boldsymbol{\omega}=\left(\omega_{x}, \omega_{y}, \omega_{z}\right)$ and the $\boldsymbol{a}$-vorticity $\Omega_{a}(\boldsymbol{a})=$ $\left(\Omega_{a}, \Omega_{b}, \Omega_{c}\right)$. From $(72)$, these are

$$
\Omega_{a}=\omega_{x}\left(\partial_{b} y \partial_{c} z-\partial_{c} y \partial_{b} z\right)+\omega_{y}\left(\partial_{b} z \partial_{c} x-\partial_{c} z \partial_{b} x\right)+\omega_{z}\left(\partial_{b} x \partial_{c} y-\partial_{c} x \partial_{b} y\right),
$$

with other two equations for $\Omega_{b}$ and $\Omega_{c}$ determined cyclically.

There are three vectors (velocity, acceleration and vorticity) determined by evolution equations subject to initial conditions in each space of $\boldsymbol{x}$ and $\boldsymbol{a}$ coordinates. Transformation relations of the three vectors suffice to determine the nine matrix elemets $\partial x^{k} / \partial a^{l}$ locally. Thus, the transformation between the Lagrangian $\boldsymbol{a}$ space and Eulerian $\boldsymbol{x}(\boldsymbol{a})$ space is determined uniquely. In this sense, the equation of the vorticity is essential for the uniqueness of the transformation between Lagrangian and Eulerian coordinates.

\subsection{Two linking vortices (an example)}

As an example of flows of non-zero helicity, we consider a particular flow field induced by tangling of two vortices, i.e. a rectilinear line-vortex L linked with a vortex ring $\mathrm{R}$ of infinitesimal vortex-cores, whose cross-sections are both circular and strengths are $\gamma_{L}$ and $\gamma_{R}$ respectively. The helicity $H$ is immediately found as $2 \gamma_{L} \gamma_{R}$ (see below), hence it is non-zero. Therefore, this flow field cannot be given a representation of a Clebsch-type form.

We consider a flow of an incompressible fluid of density $\rho_{0}$ in a cylindrical coordinate frame $(x, r, \phi)$. Suppose that we have a rectilinear line-vortex $\mathrm{L}$ of strength $\gamma_{L}(>0)$ coinciding with the $x$-axis (axis of cylindrical symmetry) and a vortex ring $\mathrm{R}$ of strength $\gamma_{R}(>0)$ which coincides temporarily with a 
circle of radius $R$ in the plane $x=0$ centered at the origin. By symmetry consideration, it is readily seen for this initial configuration that the vortex ring $R$ simply translates parallel to the $x$ axis with a constant velocity $U$ (say) without change of its form and its radius (apart from rotaional motion around the $x$ axis without changing its form), while the rectilinear vortex $L$ stays at the same position without change. With respect to this coordinate frame, the velocity field (at the initial instant) of the rectilinear vortex $\mathrm{L}$ is represented by a vector potential $\Psi_{L}=(F(r), 0,0)$, while that of the vortex ring $\mathrm{R}$ is given by $\Psi_{R}=(0,0, G(x, r))$. Explicit expressions of velocity are $\boldsymbol{v}_{L}=\nabla \times \Psi_{L}$ and $\boldsymbol{v}_{R}=\nabla \times \Psi_{R}$ respectively, and

$$
\begin{aligned}
& \boldsymbol{v}_{L}=\left(0,0,-\partial_{r} F\right), \quad F=-\frac{\gamma_{L}}{2 \pi} \log r, \\
& \boldsymbol{v}_{R}=\left(r^{-1} \partial_{r}(r G),-\partial_{x} G, 0\right), \\
& \quad G(x, r)=\frac{\gamma_{R}}{4 \pi} R \int_{0}^{2 \pi} \frac{\cos \phi \mathrm{d} \phi}{\sqrt{x^{2}+r^{2}-2 r R \cos \phi+R^{2}}} .
\end{aligned}
$$

Their vorticities are

$$
\boldsymbol{\omega}_{L}=\left(\gamma_{L} \delta(y) \delta(z), 0,0\right), \quad \boldsymbol{\omega}_{R}=\left(0,0, \gamma_{R} \delta(r-R) \delta(x)\right)
$$

where $\delta$ is the delta function, and $(y, z)$ denotes the cartesian coordinates in the plane perpendicular to the $x$-axis. Hence, the helicity is given by

$$
H=\int_{\mathcal{R}^{3}}\left[\boldsymbol{v}_{L} \cdot \boldsymbol{\omega}_{R}+\boldsymbol{v}_{R} \cdot \boldsymbol{\omega}_{L}\right] \mathrm{d}^{3} \boldsymbol{x}
$$

By elementary calculus, we find that the integral of each term gives the same value $\gamma_{L} \gamma_{R}$. Thus we obtain non-zero helicity $H=2 \gamma_{L} \gamma_{R}$ (Moffatt 1969).

The potential $\boldsymbol{B}=\nabla \times A$ can be determined in the following way. In reference to the coordinate frame moving with the vortex ring $\mathrm{R}$, the velocity field is steady (and the vorticity too). In this frame, a uniform velocity $(-U, 0,0)$ is added, and total velocity is given by

$$
\boldsymbol{w}=\boldsymbol{v}_{L}+\boldsymbol{v}_{R}+(-U, 0,0)=\nabla \times \chi \equiv \boldsymbol{v}(\boldsymbol{x})=(u, v, w),
$$

where $\chi=\left(F(r)-\frac{1}{2} U r, 0, G(x, r)\right)=\chi(\boldsymbol{x})$, independent of $t$. In the equation (103): $\rho_{0} \boldsymbol{v}=E_{W}[\boldsymbol{B}]+\boldsymbol{\omega} \times \boldsymbol{B}$, the vorticity $\boldsymbol{\omega}$ is nonzero only on the rectilinear line $\mathrm{L}$ and the circular curve $\mathrm{R}$, and vanishes $(\boldsymbol{\omega}=0)$ at all other points. At points neither on $\mathrm{L}$ nor on $\mathrm{R}$, we have the equation:

$$
\rho_{0} \boldsymbol{v}=E_{W}[\boldsymbol{B}], \quad E_{W}[\boldsymbol{B}]=\partial_{t} \boldsymbol{B}+\nabla \times(\boldsymbol{B} \times \boldsymbol{v}) .
$$

Let us seek a general solution to this equation for a given velocity field $\boldsymbol{v}(\boldsymbol{x})$. Suppose that we have a particle-path solution $\boldsymbol{x}=\boldsymbol{X}(\boldsymbol{a}, t)$ satisfying $\partial_{t} \boldsymbol{X}(\boldsymbol{a}, t)=\boldsymbol{v}(\boldsymbol{X})$, where $\mathrm{D}_{t} \boldsymbol{a}=0$ for $\boldsymbol{a}=\boldsymbol{a}(\boldsymbol{x}, t)$ and $\boldsymbol{a}(\boldsymbol{x}, 0)=\boldsymbol{x}$. Next, 
we introduce a particular field $\boldsymbol{B}_{*}(\boldsymbol{a}, t)$ defined by

$$
\boldsymbol{B}_{*}(\boldsymbol{a}, t) \equiv\left(\boldsymbol{B}_{*}(\boldsymbol{a}, 0) \cdot \frac{\partial}{\partial \boldsymbol{a}}\right) \boldsymbol{X}(\boldsymbol{a}, t),
$$

which satisfies the equation: $E_{W}\left[\boldsymbol{B}_{*}\right]=\partial_{t} \boldsymbol{B}_{*}+\nabla \times\left(\boldsymbol{B}_{*} \times \boldsymbol{v}\right)=0$ (Cauchy's solution) for an arbitrary initial field $\boldsymbol{B}_{*}(\boldsymbol{x}, 0)$. A general solution to the equation (108) is given by

$$
\boldsymbol{B}(\boldsymbol{x}, t)=\boldsymbol{B}_{*}(\boldsymbol{a}, t)+\boldsymbol{B}_{0}(\boldsymbol{x}),
$$

where $\boldsymbol{B}_{0}(\boldsymbol{x})$ is a time-independent field and a particular solution to (108). In fact, substituting (109) into (108), we have $\rho_{0} \nabla \times \chi=\nabla \times\left(\boldsymbol{B}_{0} \times \boldsymbol{v}\right)$, in view of (107) and $E_{W}\left[\boldsymbol{B}_{*}\right]=0$. Thus, we obtain

$$
\boldsymbol{B}_{0} \times \boldsymbol{v}=\nabla \psi+\rho_{0} \chi \quad(\equiv \boldsymbol{Q}),
$$

where $\psi$ is a scalar function satisfying $\boldsymbol{v} \cdot \boldsymbol{Q}=(\boldsymbol{v} \cdot \nabla) \psi+\rho_{0} \boldsymbol{v} \cdot \chi=0$. Then, the solution $\boldsymbol{B}_{0}$ is given by

$$
\boldsymbol{B}_{0}=\left(0,-Q_{\phi}, Q_{r}\right) / u, \quad \boldsymbol{Q}=\left(Q_{x}, Q_{r}, Q_{\phi}\right) .
$$

\section{Summary and discussion}

Following the scenario of the gauge principle of field theory, it is found that the variational principle of fluid motions can be reformulated successfully in terms of a covariant derivative and Lagrangians defined appropriately. The Lagrangians are determined such that a gauge invariance is satisfied under local Galilean transformations including the rotational symmetry. The fluid material is characterized thermodynamically in terms of mass density and entropy density. In addition, local rotation (i.e. vorticity) of the material is also taken into account by a newly-introduced Lagrangian $\Lambda_{A}$. A gauge-covariant derivative $\nabla_{t}$ was defined in the present formulation including the rotational symmetry (it was $\mathrm{D}_{t}$ up to $\S 5$ for the translation symmetry alone). Galilean invariance requires that the covariant derivative should be the convective time derivative following the motion of the background material, i.e. the so-called Lagrange derivative. The covariant derivative is an essential building block of the gauge theory. Applying $\nabla_{t}$ to the position coordinate $\boldsymbol{x}(\boldsymbol{a}, t)$ of a fluid particle $\boldsymbol{a}$, we obtain the particle velocity $\boldsymbol{v}$. It is found that the vorticity $\boldsymbol{\omega}$ is in fact a gauge field associated with the rotational symmetry. Lastly, an example of flow with non-vanishing helicity is presented together with a general solution of the vector potential for the rotational part.

The variational principle (i.e. the action principle) in the space of particle coordinate $\boldsymbol{a}$ results in the Euler-Lagrange equation. This yields the Noether 
thorem, i.e. conservation equations of energy-momentum. The equations thus obtained are the Lagrangian form of the equation of motion and energy equation, which are transformed to Euler's equation of motion and the conservation equation of total energy $\frac{1}{2} v^{2}+\epsilon$ in Eulerian space.

The variational principle with respect to Eulerian space coordinate $\boldsymbol{x}$ results in (i) the expression of velocity $\boldsymbol{v}$ in terms of gauge potentials, (ii) an integral describing a potential part of the velocity, (iii) the equation of continuity, (iv) the entropy equation. In addition to these results obtained in the previous case of translation symmetry alone, new results in the present formulation are as follows. (v) The velocity $\boldsymbol{v}$ includes a new rotational term $\boldsymbol{w}$, (vi) the vorticity equation (93) is deduced from the variational principle and (vii) the equation (101) is derived for the rotational component $\boldsymbol{w}$. It is noteworthy that the new Lagrangian $\Lambda_{A}$ of (86) yields a source term for the helicity defined by (104).

Transformation from the Lagrangian $\boldsymbol{a}$ space to Eulerian $\boldsymbol{x}$ is determined locally by nine components of the matrix $\partial x^{k} / \partial a^{l}$. However, in the solution obtained previously (Kambe 2007) without taking account of rotational symmetry, we had only six relations for the velocity (48) and acceleration (51) including the matrix element $\partial x^{k} / \partial a^{l}$. Both of the equations are invariant under rotational transformations of a displacement vector $\Delta \boldsymbol{X}=(\Delta X, \Delta Y, \Delta Z)$, so that the vector $\Delta \boldsymbol{X}$ is not uniquely determined. In the improved formulation taking account of the rotational symmetry with three additional equations from (72), there are three vectors (velocity, acceleration and vorticity) determined by evolution equations subject to initial conditions in each space of $\boldsymbol{x}$ and $\boldsymbol{a}$. Transformation relations of the three vectors suffice to determine the nine matrix elements $\partial x^{k} / \partial a^{l}$ locally. Thus, the transformation between the Lagrangian $\boldsymbol{a}$ space and Eulerian $\boldsymbol{x}(\boldsymbol{a})$ space is determined uniquely. In this sense, the equation (105) for the vorticity is essential to the uniqueness of the transformation between Lagrangian and Eulerian coordinates.

Accordingly, the present variational formulation is self-consistent and comprehensively describes flows of an ideal fluid.

\section{Acknowledgment}

The author is grateful to Professor Antti Niemi for discussions on the ChernSimons term of the gauge theory, and also deeply appreciates the careful comments by Professor Alex Craik on the original manuscript, which have led to significant improvement of the expressions. He is also much obliged to the referees and the editor for the comments to improve the manuscript. 


\section{Appendix: Background of the theory}

This appendix reviews the background of the theory and describes the scenario of the gauge principle in a physical system.

\section{A Gauge invariances}

In the theory of electromagnetism, it is well-known that there is an invariance under a gauge transformation of electromagnetic potentials consisting of a scalar potential $\phi$ and a vector potential $\boldsymbol{A}$. The electric field $\boldsymbol{E}$ and magnetic field $\boldsymbol{B}$ are represented as $\boldsymbol{E}=-\nabla \phi-(1 / c)(\partial \boldsymbol{A} / \partial t)$, and $\boldsymbol{B}=\nabla \times \boldsymbol{A}$, where $c$ is the light velocity. The idea is that the fields $\boldsymbol{E}$ and $\boldsymbol{B}$ are unchanged by the following transformation: $(\phi, \boldsymbol{A}) \rightarrow\left(\phi^{\prime}, \boldsymbol{A}^{\prime}\right)$, where $\phi^{\prime}=\phi-c^{-1} \partial_{t} f$, $\boldsymbol{A}^{\prime}=\boldsymbol{A}+\nabla f$ with $f(\boldsymbol{x}, t)$ being an arbitrary differentiable scalar function of position $\boldsymbol{x}$ and time $t$.

Gauge invariance is also known in certain rotational flows of the Clebsch representation (Eckart 1960). Its velocity field is represented as (Sec.3.2):

$$
\boldsymbol{v}=\nabla \phi+s \nabla \psi
$$

where $\phi, \psi$ and $s$ are scalar functions, and $\psi$ satisfies the equation $\mathrm{D}_{t} \psi=0$. Let $\phi^{\prime}, \psi^{\prime}$ and $s^{\prime}$ be a second set giving the same velocity field $\boldsymbol{v}$, which implies the following: $s \nabla \psi-s^{\prime} \nabla \psi^{\prime}=\nabla\left(\phi^{\prime}-\phi\right)$. This relation will hold true by the relation $\phi^{\prime}-\phi=F\left(\psi, \psi^{\prime}\right)$, if $s=\partial F / \partial \psi$ and $s^{\prime}=-\partial F / \partial \psi^{\prime}$. Therefore, $s$ and $\psi$ are determined only up to such a contact transformation, and $\phi$ transforms by the addition of the generating function $F\left(\psi, \psi^{\prime}\right)$. It can be shown that the two sets of triplet give the identical flow field. Thus, there exist some freedom in the expressions of physical fields in terms of potentials.

\section{B Related aspects in quantum mechanics and relativity theory}

In quantum mechanics, Schrödinger's equation for a charged particle of mass $m$ and electromagnetic fields are invariant with respect to a gauge transformation. This is as follows. In the absence of electromagnetic fields, Schrödinger's equation for a wave function $\psi$ of a free particle $m$ is written as $S[\psi] \equiv i \hbar \partial_{t} \psi-$

$\frac{1}{2 m} p_{k}^{2} \psi=0$, where $p_{k}=-i \hbar \partial_{k}$ is the momentum operator $\left(\partial_{k}=\partial / \partial x^{k}\right.$ for $k=1,2,3)$. In the presence of electromagnetic fields, Schrödinger's equation for a particle with an electric charge $e$ is obtained by the transformation:

$$
\partial_{t} \rightarrow \partial_{t}+(e / i \hbar) A_{0}, \quad \partial_{k} \rightarrow \partial_{k}+(e / i \hbar c) A_{k}, \quad(k=1,2,3),
$$


where $\left(A_{\mu}\right)=\left(A_{0}, A_{1}, A_{2}, A_{3}\right)=\left(A_{0}, \boldsymbol{A}\right)=(-\phi, \boldsymbol{A})$ is a four-vector potential, actually a covector denoted with lower indices. A point in space-time is denoted by a four vector with upper indices, $\left(x^{\mu}\right)=\left(x^{0}, x^{1}, x^{2}, x^{3}\right)$ with $x^{0}=$ ct. Thus, we obtain the equation with electromagnetic fields:

$$
S_{A}[\psi] \equiv i \hbar \partial_{t} \psi-e \phi \psi-\frac{1}{2 m}\left[(-i \hbar)\left(\partial_{k}+\frac{e}{i \hbar c} A_{k}\right)\right]^{2} \psi=0
$$

From the four potential $A_{\mu}=(-\phi, \boldsymbol{A})$, we obtain $\boldsymbol{E}$ and $\boldsymbol{B}$.

Suppose that a wave function $\psi\left(x^{\mu}\right)$ satisfies the equation $S_{A}[\psi]=0$, and consider the following set of transformations of $\psi\left(x^{\mu}\right)$ and $A_{\mu}$ :

$$
\begin{aligned}
\psi^{\prime}\left(x^{\mu}\right) & =\exp \left[i \alpha\left(x^{\mu}\right)\right] \psi\left(x^{\mu}\right), \\
A_{k} & \rightarrow A_{k}^{\prime}=A_{k}+\partial_{k} \beta, \quad \phi \rightarrow \phi^{\prime}=\phi-c^{-1} \partial_{t} \beta,
\end{aligned}
$$

where $\alpha=(e / \hbar c) \beta$. Then, it is shown readily that the transformed function $\psi^{\prime}\left(x^{\mu}\right)$ satisfies the Schrödinger equation $S_{A^{\prime}}\left[\psi^{\prime}\right]=0$. This is the gauge invariance of the system of an electric charge in electromagnetic fields. The system is said to have a gauge symmetry.

A relativistic Lagrangian (i.e. Lorentz-invariant) is defined for fluid flows by Soper (1976), and the energy-momentum tensor $T^{\mu \nu}$ is derived from it, where $\mu, \nu=0,1,2,3$ with 0 denoting the time component. In this relativistic formulation, the continuity equation and an energy equation of fluid flows are derived from the conservation of particle numbers and an equation of energy conservation $\partial_{\nu} T^{0 \nu}=0$. Expanding the energy density $T^{00}$ and energy flux $T^{0 k}$ with respect to an infinitesimal ratio $\beta=v / c(k=1,2,3$, where $c$ is the light velocity and $v$ a representative fluid velocity), the continuity equation is obtained from the lowest order terms (equivalent to the conservation of particle number), while nonrelativistic energy conservation is obtained from the next order terms proportional to $\beta^{2}$. The entropy equation is another form of the energy conservation.

\section{Brief scenario of gauge principle}

In the gauge theory of particle physics (Weinberg 1995; Frankel 1997), a freeparticle Lagrangian $\Lambda_{\text {free }}[\psi]$ is defined first for the wave function $\psi\left(x^{\mu}\right)$ of a particle with an electric charge. Let us consider the following gauge transformation: $\psi \mapsto e^{i \alpha} \psi$. If $\Lambda_{\text {free }}$ is invariant under the transformation when $\alpha$ is a constant, it is said that $\Lambda_{\text {free }}$ has a global gauge invariance. In spite of this, it often happens that $\Lambda_{\text {free }}$ is not invariant for a function $\alpha=\alpha(x)$, i.e. $\Lambda_{\text {free }}$ 
is not gauge-invariant locally. In this circumstance, it is instrumental to introduce a new field in order to acquire local gauge invariance. If the new field (a gauge field) is chosen appropriately, local gauge invariance can be recovered.

In the section $\mathrm{B}$, the local gauge-invariance was acquired by replacing $\partial_{\mu}$ with

$$
\mathrm{D}_{\mu}=\partial_{\mu}+\mathcal{A}_{\mu}
$$

(see (B.1)), where $\mathcal{A}_{\mu}=(e / i \hbar c) A_{\mu}$, and $A_{\mu}(x)$ is the electromagnetic potential (and termed a connection form in mathematics). The operator $\mathrm{D}_{\mu}$ is called the covariant derivative.

Thus, when the original Lagrangian is not locally gauge invariant, the principle of local gauge invariance requires a new gauge field to be introduced in order to acquire local gauge invariance, and the Lagrangian is to be altered by replacing the partial derivative with the covariant derivative including the gauge field. This is the Weyl's gauge principle.

In mathematical terms, suppose that we have a group $\mathcal{G}$ of transformations and an element $g(x) \in \mathcal{G}$ (for $x \in M$ with $M$ a space where $\psi$ is defined) and that the wave function $\psi(x)$ is transformed as $\psi^{\prime}=g(x) \psi$. In the previous example, $g(x)=e^{i \alpha(x)}$ and the group is $\mathcal{G}=U(1)$. Introducing the gauge field $A$ allows us to define a covariant derivative $\mathrm{D}=\partial+A$ as a generalization of the partial derivative $\partial$ that transforms as $g \mathrm{D}=g(\partial+A)=\left(\partial^{\prime}+A^{\prime}\right) g$. If we operates the right hand side on $\psi$, we obtain $\left(\partial^{\prime}+A^{\prime}\right) g \psi=\left(\partial^{\prime}+A^{\prime}\right) \psi^{\prime}=\mathrm{D}^{\prime} \psi^{\prime}$ where $\mathrm{D}^{\prime}=\partial^{\prime}+A^{\prime}$. Thus, we obtain $\mathrm{D}^{\prime} \psi^{\prime}=g \mathrm{D} \psi$, showing that $\mathrm{D} \psi$ transforms in the same way as $\psi$ itself.

In dynamical systems which evolve with the time $t$, such as the present case of fluid flows, replacement is to be made only for the $t$ derivative: $\partial_{t} \rightarrow \mathrm{D}_{t}=$ $\partial_{t}+A(x)$.

\section{References}

[1] Aitchison IJR and Hey AJG 1982. Gauge Theories in Particle Physics. Adam Hilger (Bristol).

[2] Arnold VI and Khesin B 1998. Topological methods in hydrodynamics, Springer.

[3] Bretherton FP 1970. A note on Hamilton's principle for perfect fluids. J. Fluid Mech. 44, 19-31.

[4] Chern SS 1979. Complex manifolds without potential theory, 2nd ed., Springer-Verlag (Berlin).

[5] Clebsch A 1859. J. Reine Angew. Math., 56, 1.

[6] Desser S, Jackiw R and Templeton S 1982. Topologically massive gauge theories, Annals of Physics 140, 372 - 411. 
[7] Eckart C 1960. Phys. Fluids, 3, 421; Eckart C 1938. Phys. Rev., 54, 920.

[8] Frankel T 1997. The Geometry of Physics An Introduction. CUP.

[9] Herivel JW 1955. The derivation of the equations of motion of an ideal fluid by Hamilton's principle. Proc. Cambridge Phil. Soc. 51, 344-349.

[10] Jackiw R 2002. Lectures on Fluid Dynamics, Springer.

[11] Jackiw R 1985. Chern-Simons terms and cocycles in physics and mathematics, in Fradkin Festschrift, Adam Hilger (Bristol).

[12] Kambe T 2003a. Gauge principle for flows of a perfect fluid. Fluid Dyn. Res. 32, 193-199.

[13] Kambe T 2003b. Gauge principle and variational formulation for flows of an ideal fluid. Acta Mechanica Sinica 19, 437-452.

[14] Kambe T 2007. Gauge principle and variational formulation for ideal fluids with reference to translation symmetry, Fluid Dyn. Res. 39.

[15] Lamb H 1932. Hydrodynamics. Cambridge University Press.

[16] Landau LD and Lifshitz EM 1976. Mechanics (3rd ed). Pergamon Press.

[17] Landau LD and Lifshitz EM 1987. Fluid Mechanics (2nd ed). Pergamon.

[18] Lin CC 1963. Hydrodynamics of helium II, in Liquid Helium. Proc. Int. Sch. Phys. "Enrico Fermi" XXI (NY: Academic Press), 93-146.

[19] Moffatt HK 1969. J. Fluid Mech. 35, 117.

[20] Noether E 1918. Invariante Variationsproblem. Klg-Ges. Wiss. Nach. Göttingen, Math. Physik Kl, 2, 235.

[21] Salmon R 1988. Ann.Rev.Fluid Mech. 20, 225-256.

[22] Seliger RL and Whitham, GB 1968. Variational principle in continuum mechanics. Proc. Roy. Soc.A 305, 1-25.

[23] Serrin J 1959. Mathematical principles of classical fluid mechanics. Encyclopedia of Physics (eds: S.Flügge and C.Truesdell), Springer, 125-263.

[24] Soper DE 1976. Classical Field Theory, John Wiley and Sons.

[25] Taub AH 1954. Phys. Rev. 94, 1468.

[26] Weinberg S 1995. The Quantum Theory of Fields, Vol. I (1995) and II (1996). Cambridge University Press. 